\title{
PULLBACK ATTRACTORS FOR A NON-AUTONOMOUS INTEGRO-DIFFERENTIAL EQUATION WITH MEMORY IN SOME UNBOUNDED DOMAINS
}

\author{
María Anguiano, Tomás Caraballo and José Real \\ Dpto. Ecuaciones Diferenciales y Análisis Numérico, Universidad de Sevilla, Apdo. de Correos 1160 \\ Sevilla, 41080, Spain \\ anguiano@us.es,caraball@us.es,jreal@us.es \\ José Valero \\ Centro de Investigación Operativa, Universidad Miguel Hernández, Avda. de la Universidad, s/n \\ Elche, 03202, Spain \\ jvalero@umh.es
}

\begin{abstract}
The main aim of this paper is to analyse the asymptotic behaviour of a non-autonomous integrodifferential parabolic equation of diffusion type with a memory term, expressed by convolution integrals involving infinite delays, in an unbounded domain. The assumptions imposed do not ensure uniqueness of solutions of the corresponding initial value problems. The theory of set-valued non-autonomous dynamical systems is applied to prove the existence of pullback attractors for our model. To do this, we first analyse an abstract version of the equation.
\end{abstract}

Keywords: Delayed reaction-diffusion equations; Integro-differential equations with memory; Pullback attractors; Multivalued non-autonomous dynamical systems; Asymptotic behavior.

\section{OBITUARY}

While this paper was in the referee procedure, our colleague and much better friend José Real sadly passed away when he was sixty years old. He was an excellent mathematician and we very much enjoyed learning Maths from and with him. However, being this important, what we will most miss is his friendship, his sense of humor, and the good moments of our lives that we shared with him. He will not only be present in our memories for the rest of our lives, but also in the bottom of our hearts.

\section{Introduction}

In all the paper we assume that $\Omega \subset \mathbb{R}^{N}$ is a given nonempty open set, not necessarily bounded, such that the Poincaré inequality is satisfied in $\Omega$, i.e., there exists a constant $\lambda_{1}>0$ such that

$$
\int_{\Omega}|u(x)|^{2} d x \leq \lambda_{1}^{-1} \int_{\Omega}|\nabla u(x)|^{2} d x, \forall u \in H_{0}^{1}(\Omega) .
$$

Let us consider the following non-autonomous reaction-diffusion equation with memory

$$
\frac{\partial u}{\partial t}-\Delta u+\int_{-\infty}^{t} \gamma(t-s) \Delta u(x, s) d s+g(x, t, u(x, t))=f(t),
$$


with Dirichlet boundary condition, where $x$ belongs to $\Omega, f$ and $\gamma$ satisfy suitable assumptions ( $\gamma$ can be given in a standard way as $\gamma(t)=-\gamma_{0} e^{-d_{0} t}$ (see [Chepyzhov et al., 2006a] and [Chepyzhov et al., 2006b]) with $d_{0}>0$ and $\left.\gamma_{0}>0\right)$. The function $g:(x, t) \in \Omega \times \mathbb{R} \mapsto g(x, t, r) \in \mathbb{R}$ is measurable for all $r \in \mathbb{R}$, with $g(x, t, \cdot) \in C(\mathbb{R})$ for a.e. $(x, t) \in \Omega \times \mathbb{R}$, and satisfies that there exist positive constants $\eta, \rho$, and $p \geq 2$, and positive functions $\delta_{i} \in L_{l o c}^{1}\left(\mathbb{R} ; L^{1}(\Omega)\right), i=1,2$, such that a.e. $(x, t) \in \Omega \times \mathbb{R}$,

$$
g(x, t, r) r \geq \eta|r|^{p}-\delta_{1}(x, t),
$$

and

$$
|g(x, t, r)|^{q} \leq \rho|r|^{p}+\delta_{2}(x, t),
$$

for all $r \in \mathbb{R}$, where $q$ is the conjugate exponent of $p$, i.e. $1 / p+1 / q=1$. Many physical phenomena are better described if one considers in the equations of the model some terms which take into account the past history of the system. Although, in some situations, the contribution of the past history may not be so relevant to significantly affect the asymptotic behaviour of the problem, in certain models, such as those describing high viscosity liquids at low temperatures, or the thermomechanical behaviour of polymers (see [Fabrizio \& Morro, 1992] and [Renardy et al., 1987]) the past history plays a nontrivial role.

On some occasions, some phenomena are modelled by nonlinear evolutionary equations which do not take into account all the relevant information of the real systems. Instead some neglected quantities can be modelled as an external force which in general becomes time-dependent. For this reason, non-autonomous systems are of great importance and interest.

The asymptotic behaviour of equations with memory has been much studied in recent years. The asymptotic behavior of a stochastic version of (2), with an additive noise and with conditions ensuring uniqueness of the Cauchy problem was studied in [Caraballo et al., 2008]. In [Caraballo et al., 2010], the long-time behaviour of a variant of our model in a bounded domain, with memory terms expressed by convolution integrals involving infinite delays, and by a forcing term with bounded delay, is investigated.

In [Anguiano et al., 2010, 2012] a non-autonomous reaction-diffusion equation without delay in an unbounded domain is considered in which the non-autonomous term takes values in $H^{-1}$ and the nonlinear term satisfies dissipative and growth conditions which are not sufficient to ensure the uniqueness of the Cauchy problem. Using the theory of pullback attractors for multi-valued non-autonomous dynamical systems, the asymptotic behaviour of solutions is studied.

In [Chepyzhov et al., 2006a], the relation between the global attractor and trajectory attractor for equations with memory and uniqueness of the Cauchy problem is analyzed. In [Chepyzhov et al., 2006b], the authors establish some decay properties of the semigroup generated by a linear integro-differential equation for a class of memory functions in a Hilbert space arising from heat conduction with memory. In [Chepyzhov \& Miranville, 2006], using the method of trajectory attractors, the authors present a global scheme for the construction of connected trajectory and global attractors for heat equations with linear fading memory and with nonlinear heat sources. In [Gatti et al., 2005], a reaction-diffusion equation in which the diffusion term depends on the past history of the diffusion itself is considered. The authors are able to construct a Lyapunov functional associated with the dynamical systems in an appropriate history phase space. The existence of global attractor of a class of reaction-diffusion equations with finite delay and uniqueness of the Cauchy problem is proved in [Wang \& Xu, 2003].

We extend the results of these previous papers to a non-autonomous reaction-diffusion equation with memory, in an unbounded domain, by considering a nonlinear term $g$ which not ensure uniqueness of the Cauchy problem. We construct a multivalued process associated to the problem and study the existence of pullback attractors for it.

Due to the fact that the memory term involves an infinite delay which is given by a convolution term and second-order partial derivatives, we study the existence of pullback attractors in the space $\mathcal{H}$ given by measurable functions $t \mapsto u(t) \in H_{0}^{1}(\Omega)$ with $\int_{-\infty}^{0} \int_{\Omega} e^{\lambda_{1} s}(\nabla u(s))^{2} d x d s<\infty$ such that $u(0) \in L^{2}(\Omega)$. The fact that the domain is unbounded implies that the techniques previously used in [Caraballo et al., 2010] do not work in our case.

The structure of the paper is as follows. In Section 2 we introduce an abstract non-autonomous PDE, which contains, in particular, our model. We assume that the coefficients of the abstract problem contain 
infinite delay terms and satisfy several weak conditions. Then, we prove the existence of at least one weak solution for (2). Some preliminaries on the theory of set-valued non-autonomous dynamical systems are stated in Section 3. Finally, the existence of pullback attractors for the abstract problem is proved in Section 4 and in the last section we apply these results to our problem (2).

\section{Setting of the problem. Existence of solutions}

We intend to introduce a setting to find a solution of problem (2). First, we analyse an abstract parabolic equation, which contains our problem as a particular case, and then we will cover other equations at the same time.

The spaces $L^{2}(\Omega), H_{0}^{1}(\Omega)$ and $H^{-1}(\Omega)$ are denoted by $H, V$ and $V^{\prime}$, respectively. By $|\cdot|,|\nabla \cdot|$ and $\|\cdot\|_{-1}$ we denote the norms in the spaces $H, V$ and $V^{\prime}$, respectively. We introduce the space $L^{p}(\Omega)$ with norm $|\cdot|_{p}$ for $p>2$. We denote by $(\cdot, \cdot)$ the scalar product in $H$ and by $\langle\cdot, \cdot\rangle$ either the pairing between $V^{\prime}$ and $V$ or the pairing between $L^{q}(\Omega)$ and $L^{p}(\Omega)$ indistinctly.

The space $L_{\lambda_{1}}^{2}(-\infty, T ; V)$ is formed by all functions $\psi \in L_{l o c}^{2}(-\infty, T ; V)$ such that

$$
\|\psi\|_{L_{\lambda_{1}}^{2}(-\infty, T ; V)}^{2}=\int_{-\infty}^{T} e^{\lambda_{1} s}|\nabla \psi(s)|^{2} d s<\infty .
$$

We also use the abbreviation $L_{V, \lambda_{1}}^{2}=L_{\lambda_{1}}^{2}(-\infty, 0 ; V)$. For $u \in L_{\lambda_{1}}^{2}(-\infty, T ; V)$ and $t \leq T$, we will write $u_{t}: s \in(-\infty, 0] \mapsto u(t+s)$, with $u_{t} \in L_{V, \lambda_{1}}^{2}$.

We set $\mathcal{H}=H \times L_{V, \lambda_{1}}^{2}$, which is a separable Hilbert space with the norm

$$
\|(\varphi, \xi)\|_{\mathcal{H}}^{2}=|\varphi|^{2}+\|\xi\|_{L_{V, \lambda_{1}}^{2}}^{2} .
$$

We aim to analyze the following non-autonomous evolution equation

$$
\left\{\begin{aligned}
\frac{d u}{d t}(t)-\Delta u(t) & =K\left(t, u_{t}\right)-G(t, u(t))+F(t) \text { for } t>\tau \\
u(\tau) & =u^{\tau} \\
u(s+\tau) & =\psi(s) \text { for } s<0
\end{aligned}\right.
$$

where $\tau \in \mathbb{R}, \psi \in L_{V, \lambda_{1}}^{2}, F \in L_{l o c}^{2}\left(\mathbb{R} ; V^{\prime}\right)$, and

$$
\begin{gathered}
G: \mathbb{R} \times L^{p}(\Omega) \rightarrow L^{q}(\Omega), \\
K: \mathbb{R} \times L_{V, \lambda_{1}}^{2} \rightarrow V^{\prime},
\end{gathered}
$$

are measurable mappings such that for a.e. $t \in \mathbb{R}, G(t, \cdot): L^{p}(\Omega) \rightarrow L^{q}(\Omega)$ and $K(t, \cdot): L_{V, \lambda_{1}}^{2} \rightarrow V^{\prime}$ are continuous operators.

We will assume that

$$
\langle G(t, v), v\rangle \geq \eta|v|_{p}^{p}-c_{1}(t)
$$

and

$$
|G(t, v)|_{q}^{q} \leq \rho|v|_{p}^{p}+c_{2}(t), \text { for } v \in L^{p}(\Omega),
$$

where $c_{i} \in L_{l o c}^{1}(\mathbb{R}), i=1,2$, are positive functions.

For all $n \geq 1$, we consider

$$
\Omega_{n}=\Omega \cap\left\{x \in \mathbb{R}^{N}:|x|_{\mathbb{R}^{N}}<n\right\},
$$

where $|\cdot|_{\mathbb{R}^{N}}$ denotes the Euclidean norm in $\mathbb{R}^{N}$.

Let us now state a set of assumptions which will be imposed along the paper. 
(H1) There is a $d \in(0,1)$ and a positive function $c_{3} \in L_{l o c}^{1}(\mathbb{R})$ such that

$$
4 \int_{\tau}^{t} e^{\lambda_{1} s}\left\|K\left(s, u_{s}\right)\right\|_{-1}^{2} d s \leq \int_{\tau}^{t} e^{\lambda_{1} s} c_{3}(s) d s+\frac{d}{2} \int_{-\infty}^{t} e^{\lambda_{1} s}|\nabla u(s)|^{2} d s,
$$

for all $\tau \in \mathbb{R}, t \geq \tau$, and $u \in L_{\lambda_{1}}^{2}(-\infty, t ; V)$. In addition, there exist a $k>0$ and a positive function $c_{4} \in L_{l o c}^{1}(\mathbb{R})$ for which

$$
\|K(t, \psi)\|_{-1}^{2} \leq c_{4}(t)+k\|\psi\|_{L_{V, \lambda_{1}}^{2}}^{2} \text {, for } \psi \in L_{V, \lambda_{1}}^{2} \text {, and for } t \in \mathbb{R} .
$$

(H2) For any $n \geq 1$ and any sequence $\left\{u^{m}\right\}_{m \in \mathbb{N}}$ such that $u^{m} \rightarrow u$ strongly in $L^{2}\left(\tau, T ; L^{2}\left(\Omega_{n}\right)\right)$, and $u^{m} \rightarrow u$ weakly in $L^{p}\left(\tau, T ; L^{p}(\Omega)\right)$ it follows that

$$
G\left(\cdot, u^{m}(\cdot)\right) \rightarrow G(\cdot, u(\cdot)) \text { weakly in } L^{q}\left(\tau, T ; L^{q}\left(\Omega_{n}\right)\right) .
$$

(H3) For any $T>\tau$ and any sequence $\left\{u^{m}\right\}_{m \in \mathbb{N}}$, the convergence $u^{m} \rightarrow u$ weakly in $L_{\lambda_{1}}^{2}(-\infty, T ; V)$ implies that

$$
K\left(\cdot, u_{.}^{m}\right) \rightarrow K(\cdot, u \text {. }) \text { weakly in } L^{2}\left(\tau, T ; V^{\prime}\right) .
$$

(H4) For all $t>\tau$ and any $u, v \in L_{\lambda_{1}}^{2}(-\infty, t ; V)$, we have

$$
2 \int_{\tau}^{t} e^{\lambda_{1} s}\left\|K\left(s, u_{s}\right)-K\left(s, v_{s}\right)\right\|_{-1}^{2} d s \leq \frac{b}{2} \int_{-\infty}^{t} e^{\lambda_{1} s}|\nabla u(s)-\nabla v(s)|^{2} d s,
$$

where $0<b<1$.

(H5) The operator $K$ satisfies

$$
\int_{-\infty}^{t} e^{\lambda_{1} s}\left\|K\left(s, u_{s}\right)\right\|_{-1}^{2} d s<+\infty
$$

for all $t \in \mathbb{R}$ and any $u \in L_{\lambda_{1}}^{2}(-\infty, t ; V)$.

(H6) The function $F$ satisfies

$$
\int_{-\infty}^{t} e^{\lambda_{1} s}\|F(s)\|_{-1}^{2} d s<+\infty \quad \forall t \in \mathbb{R} .
$$

Remark 2.1. If we define $G: \mathbb{R} \times L^{p}(\Omega) \rightarrow L^{q}(\Omega)$ as

$$
G(t, v)(x)=g(x, t, v(x))
$$

for $v \in L^{p}(\Omega), t \in \mathbb{R}$ and $x \in \Omega$, where $g$ is given in Section 1, then we will see in Section 5 that thanks to (3) and (4), we can deduce that $G$ is continuous in $v$ and satisfies (6), (7) and (H2), with $c_{i}(t)=\int_{\Omega} \delta_{i}(x, t) d x$, $i=1,2$.

Remark 2.2. Observe that by assumption (1) the operator $K$ can be written as $K=\sum_{i=1}^{N} \frac{\partial K_{i}}{\partial x_{i}}$, with $K_{i}$ : $\mathbb{R} \times L_{V, \lambda_{1}}^{2} \rightarrow H$ measurable mappings satisfying that for a.e. $t \in \mathbb{R}, K_{i}(t, \cdot): L_{V, \lambda_{1}}^{2} \rightarrow H$ is continuous for all $1 \leq i \leq N$, and

$$
\|K(t, \xi)\|_{-1}^{2}=\sum_{i=1}^{N}\left|K_{i}(t, \xi)\right|^{2}
$$

for all $\xi \in L_{V, \lambda_{1}}^{2}$.

Analogously, the function $F$ can be written as $F=\sum_{i=1}^{N} \frac{\partial F_{i}}{\partial x_{i}}$, with $F_{i} \in L_{l o c}^{2}(\mathbb{R} ; H)$ for all $1 \leq i \leq N$ such that

$$
\|F(t)\|_{-1}^{2}=\sum_{i=1}^{N}\left|F_{i}(t)\right|^{2} \quad \text { a.e. } t \in \mathbb{R}
$$


We state a result on the existence of solution of problem (5). First, we give the definition of weak solution.

Definition 2.1. A weak solution to the non-autonomous evolution equation (5), with initial function $\left(u^{\tau}, \psi\right) \in \mathcal{H}$, is a function $u \in L_{\text {loc }}^{2}(\mathbb{R} ; V)$ such that $u(s+\tau)=\psi(s)$ a.e. $s<0$, for any $T>\tau$ the restriction of $u$ on the interval $(\tau, T)$ is in $L^{p}\left(\tau, T ; L^{p}(\Omega)\right)$,

$$
\frac{d}{d t}(u(t), w)+(\nabla u(t), \nabla w)=\left\langle K\left(t, u_{t}\right)-G(t, u(t))+F(t), w\right\rangle \text { a.e. } t>\tau,
$$

for all $w \in L^{p}(\Omega) \cap V$, and

$$
u(\tau)=u^{\tau}
$$

Observe that by (7) and (10), if $u$ is a weak solution of (5), then $u$ has a time derivative $\frac{d u}{d t}$ in $L^{2}\left(\tau, T ; V^{\prime}\right)+L^{q}\left(\tau, T ; L^{q}(\Omega)\right)$, for all $T>\tau$, and therefore, it is well known that $u \in C([\tau,+\infty) ; H)$, and $\frac{d}{d t}|u(t)|^{2}=2\left\langle\frac{d u}{d t}, u\right\rangle$ a.e. $t>\tau$. Hence, it satisfies the energy equality

$$
\frac{d}{d t}|u(t)|^{2}+2|\nabla u(t)|^{2}=2\left\langle K\left(t, u_{t}\right)-G(t, u(t))+F(t), u(t)\right\rangle \quad \text { a.e. } t>\tau .
$$

We will use the notation $u\left(\cdot ; \tau,\left(u^{\tau}, \psi\right)\right)$ to denote a weak solution of $(5)$, but we will simply write $u(\cdot)$ when no confusion is possible. The following result will be used in the proof of the main result of this paper.

Lemma 1. Under conditions (6), (7) and (H1), every weak solution u of (5) satisfies the estimates

$$
|u(t)|^{2} \leq e^{-\lambda_{1}(t-\tau)}\left\|\left(u^{\tau}, \psi\right)\right\|_{\mathcal{H}}^{2}+\int_{\tau}^{t} e^{-\lambda_{1}(t-s)} c(s) d s+4 \int_{\tau}^{t} e^{-\lambda_{1}(t-s)}\|F(s)\|_{-1}^{2} d s,
$$

and

$$
(1-d)\left\|u_{t}\right\|_{L_{V, \lambda_{1}}^{2}}^{2} \leq 2 e^{-\lambda_{1}(t-\tau)}\left\|\left(u^{\tau}, \psi\right)\right\|_{\mathcal{H}}^{2}+2 \int_{\tau}^{t} e^{-\lambda_{1}(t-s)} c(s) d s+8 \int_{\tau}^{t} e^{-\lambda_{1}(t-s)}\|F(s)\|_{-1}^{2} d s,
$$

for all $t \geq \tau$, where $c(s)=2 c_{1}(s)+c_{3}(s)$.

Proof. Using (1), (6) and (21) we have

$$
\frac{d}{d t}|u(t)|^{2}+\lambda_{1}|u(t)|^{2}+|\nabla u(t)|^{2}+2 \eta|u(t)|_{p}^{p} \leq 4\left\|K\left(t, u_{t}\right)\right\|_{-1}^{2}+\frac{1}{2}|\nabla u(t)|^{2}+2 c_{1}(t)+4\|F(t)\|_{-1}^{2} .
$$

Multiplying by $e^{\lambda_{1} t}$ and integrating between $\tau$ and $t$, we obtain

$$
\begin{aligned}
|u(t)|^{2}+\frac{1}{2} \int_{\tau}^{t} e^{-\lambda_{1}(t-s)}|\nabla u(s)|^{2} d s \leq & e^{-\lambda_{1}(t-\tau)}|u(\tau)|^{2}+4 \int_{\tau}^{t} e^{-\lambda_{1}(t-s)}\left\|K\left(s, u_{s}\right)\right\|_{-1}^{2} d s \\
& +2 \int_{\tau}^{t} e^{-\lambda_{1}(t-s)} c_{1}(s) d s+4 \int_{\tau}^{t} e^{-\lambda_{1}(t-s)}\|F(s)\|_{-1}^{2} d s .
\end{aligned}
$$

By (9), we have

$$
\begin{aligned}
4 \int_{\tau}^{t} e^{-\lambda_{1}(t-s)}\left\|K\left(s, u_{s}\right)\right\|_{-1}^{2} d s \leq & \int_{\tau}^{t} e^{-\lambda_{1}(t-s)} c_{3}(s) d s+\frac{d}{2} \int_{-\infty}^{\tau} e^{-\lambda_{1}(t-s)}|\nabla u(s)|^{2} d s \\
& +\frac{d}{2} \int_{\tau}^{t} e^{-\lambda_{1}(t-s)}|\nabla u(s)|^{2} d s \\
= & \int_{\tau}^{t} e^{-\lambda_{1}(t-s)} c_{3}(s) d s+\frac{d}{2} e^{-\lambda_{1}(t-\tau)}\|\psi\|_{L_{V, \lambda_{1}}^{2}}^{2} \\
& +\frac{d}{2} \int_{\tau}^{t} e^{-\lambda_{1}(t-s)}|\nabla u(s)|^{2} d s
\end{aligned}
$$


and thus

$$
\begin{gathered}
|u(t)|^{2}+\frac{1-d}{2} \int_{\tau}^{t} e^{-\lambda_{1}(t-s)}|\nabla u(s)|^{2} d s \leq e^{-\lambda_{1}(t-\tau)}\left(|u(\tau)|^{2}+\frac{d}{2}\|\psi\|_{L_{V, \lambda_{1}}^{2}}^{2}\right)+\int_{\tau}^{t} e^{-\lambda_{1}(t-s)} c(s) d s \\
+4 \int_{\tau}^{t} e^{-\lambda_{1}(t-s)}\|F(s)\|_{-1}^{2} d s
\end{gathered}
$$

for $t \geq \tau$, where $c=2 c_{1}+c_{3}$. In particular, we have proved (22).

On the other hand, for any $t \geq \tau$,

$$
\begin{aligned}
\left\|u_{t}\right\|_{L_{V, \lambda_{1}}^{2}}^{2} & =\int_{-\infty}^{\tau-t} e^{\lambda_{1} s}|\nabla \psi(t+s-\tau)|^{2} d s+\int_{\tau-t}^{0} e^{\lambda_{1} s}|\nabla u(t+s)|^{2} d s \\
& =e^{-\lambda_{1}(t-\tau)}\|\psi\|_{L_{V, \lambda_{1}}^{2}}^{2}+\int_{\tau}^{t} e^{-\lambda_{1}(t-s)}|\nabla u(s)|^{2} d s .
\end{aligned}
$$

From this equality and (25), we obtain (23).

Corollary 2.1. Under conditions (6), (7) and (H1), for every bounded set $B$ of $\mathcal{H}$, and for any $T>\tau$, there exists a positive constant $C=C(T, \tau, B)$ such that, for every weak solution $u=u\left(\cdot ; \tau,\left(u^{\tau}, \psi\right)\right)$ of $(5)$ corresponding to the initial data $\left(u^{\tau}, \psi\right) \in B$, we have

$$
\|u\|_{L^{p}\left(\tau, T ; L^{p}(\Omega)\right)}+\|u\|_{L^{2}(\tau, T ; V)} \leq C, \quad \forall\left(u^{\tau}, \psi\right) \in B .
$$

Proof. From (21) we obtain

$$
|u(T)|^{2}+2 \int_{\tau}^{T}|\nabla u(t)|^{2} d t+2 \int_{\tau}^{T}\langle G(t, u(t)), u(t)\rangle d t \leq\left|u^{\tau}\right|^{2}+2 \int_{\tau}^{T}\left(\left\|K\left(t, u_{t}\right)\right\|_{-1}^{2}+\|F(t)\|_{-1}^{2}\right) d t,
$$

and therefore, from (6) and (10) we deduce that

$$
\begin{aligned}
\int_{\tau}^{T}|\nabla u(t)|^{2} d t+\eta \int_{\tau}^{T}|u(t)|_{p}^{p} d t \leq & \frac{1}{2}\left\|\left(u^{\tau}, \psi\right)\right\|_{\mathcal{H}}^{2}+k \int_{\tau}^{T}\left\|u_{t}\right\|_{L_{V, \lambda_{1}}^{2}}^{2} d t \\
& +\int_{\tau}^{T}\left(c_{1}(t)+c_{4}(t)+\|F(t)\|_{-1}^{2}\right) d t
\end{aligned}
$$

Now, observe that from (23) we have in particular that

$$
\left\|u_{t}\right\|_{L_{V, \lambda_{1}}^{2}}^{2} \leq \frac{2}{1-d}\left(\left\|\left(u^{\tau}, \psi\right)\right\|_{\mathcal{H}}^{2}+\int_{\tau}^{T}\left(c(t)+4\|F(t)\|_{-1}^{2}\right) d t\right)
$$

for all $\tau \leq t \leq T$.

From this inequality and (27), we deduce (26).

Now, we formulate the main theorem of this section.

Theorem 1. Assume conditions (6), (7) and (H1)-(H3). Then, for every $\left(u^{\tau}, \psi\right) \in \mathcal{H}$, there exists at least one weak solution $u\left(\cdot ; \tau,\left(u^{\tau}, \psi\right)\right)$ to problem (5).

Proof. Consider a Hilbert basis $\left\{w_{j}: j \geq 1\right\} \subset V \cap L^{p}(\Omega)$ of $H$ such that the vector space spanned by $\left\{w_{j}: j \geq 1\right\}$ is dense in $V \cap L^{p}(\Omega)$. Let us denote by $V_{m}=\left[w_{1}, . ., w_{m}\right]$ the vector space spanned by $\left\{w_{j}: m \geq j \geq 1\right\}$, and $P_{V_{m}}^{H}: H \rightarrow V_{m}$ the projector given by $P_{V_{m}}^{H} u=\sum_{j=1}^{m}\left(u, w_{j}\right) w_{j}$. We will also denote $P_{V_{m}}^{V}: V \rightarrow V_{m}$ the orthogonal projector with respect to the norm in $V$. 
Finally, define $u^{m}(t)=\sum_{j=1}^{m} \gamma_{m j}(t) w_{j}$, where

$$
\left\{\begin{aligned}
\frac{d}{d t}\left(u^{m}(t), w_{j}\right)= & -\left(\nabla u^{m}(t), \nabla w_{j}\right)+\left\langle K\left(t, u_{t}^{m}\right), w_{j}\right\rangle \\
& -\left\langle G\left(t, u^{m}(t)\right), w_{j}\right\rangle+\left\langle F(t), w_{j}\right\rangle, \text { a.e. } t>\tau, \text { for all } 1 \leq j \leq m, \\
u^{m}(\tau)= & P_{V_{m}}^{H} u^{\tau}, \\
u^{m}(s+\tau)= & P_{V_{m}}^{V} \psi(s) \text { for } s<0 .
\end{aligned}\right.
$$

The fact that problem (28) has a local solution can be obtained as a consequence of Theorem 1.1, page 36, in [Hino et al., 1991]. The fact that this local solution is global can be deduced from uniform estimates that can be obtained reasoning similarly to the proofs of Lemma 1 and Corollary 2.1. These estimates also give that

$$
\left\{u^{m}\right\} \text { is bounded in } L_{\lambda_{1}}^{2}(-\infty, T ; V) \cap L^{p}\left(\tau, T ; L^{p}(\Omega)\right) \cap L^{2}(\tau, T ; V) \cap C([\tau, T] ; H),
$$

for all $T>\tau$. We can conclude that there exists a subsequence of solutions of the Galerkin approximations, denoted also by $\left\{u^{m}\right\}_{m \in \mathbb{N}}$, such that, for some $u$, and all $T>\tau$,

$$
\begin{gathered}
u^{m} \stackrel{*}{\rightarrow} u \text { weakly star in } L^{\infty}(\tau, T ; H), \\
u^{m} \rightarrow u \text { weakly in } L_{\lambda_{1}}^{2}(-\infty, T ; V), L^{p}\left(\tau, T ; L^{p}(\Omega)\right) \text { and } L^{2}(\tau, T ; V) .
\end{gathered}
$$

Evidently, then in particular

$$
\Delta u^{m} \rightarrow \Delta u \text { weakly in } L^{2}\left(\tau, T ; V^{\prime}\right)
$$

and, by condition (12),

$$
K\left(\cdot, u_{.}^{m}\right) \rightarrow K(\cdot, u .) \text { weakly in } L^{2}\left(\tau, T ; V^{\prime}\right) .
$$

Also, observe that by Lebesgue's dominated convergence Theorem, we have that $P_{V_{m}}^{V} \psi$ converges to $\psi$ in $L_{\lambda_{1}}^{2}(-\infty, 0 ; V)$, and therefore,

$$
u(s+\tau)=\psi(s) \text { a.e. } s<0 .
$$

Now we assume that we have proved that for all $T>\tau$ and any $n \geq 1$,

$$
u^{m} \rightarrow u \text { strongly in } L^{2}\left(\tau, T ; L^{2}\left(\Omega_{n}\right)\right),
$$

where $\Omega_{n}$ is defined in (8). For the sake of clarity, we postpone the proof of (31) to Lemma 2 below.

Then, by condition (11), we have

$$
G\left(\cdot, u^{m}(\cdot)\right) \rightarrow G(\cdot, u(\cdot)) \text { weakly in } L^{q}\left(\tau, T ; L^{q}\left(\Omega_{n}\right)\right) .
$$

On the other hand, by (7) and (29), we obtain that $G\left(\cdot, u^{m}\right)$ is bounded in $L^{q}\left(\tau, T ; L^{q}(\Omega)\right)$. Then, for every subsequence $\left\{u^{m^{\prime}}\right\}_{m^{\prime} \in \mathbb{N}}$ of the sequence $\left\{u^{m}\right\}_{m \in \mathbb{N}}$ satisfying (30), there exists a subsequence $\left\{u^{m^{\prime \prime}}\right\}_{m^{\prime} \in \mathbb{N}} \subset$ $\left\{u^{m^{\prime}}\right\}_{m^{\prime} \in \mathbb{N}}$, such that,

$$
G\left(\cdot, u^{m^{\prime \prime}}(\cdot)\right) \rightarrow \chi \text { weakly in } L^{q}\left(\tau, T ; L^{q}(\Omega)\right),
$$

in particular,

$$
G\left(\cdot, u^{m^{\prime \prime}}(\cdot)\right) \rightarrow \chi_{\Omega_{\Omega_{n} \times(\tau, T)}} \text { weakly in } L^{q}\left(\tau, T ; L^{q}\left(\Omega_{n}\right)\right) .
$$

Taking into account (32) and the uniqueness of the weak limit, we have

$$
\chi=G(\cdot, u(\cdot)) \text { a.e. in } \Omega_{n} \times(\tau, T) \quad \forall n \geq 1,
$$

and thus, in light of $\cup_{n=1}^{\infty} \Omega_{n}=\Omega$, we obtain

$$
\chi=G(\cdot, u(\cdot)) \text { a.e. in } \Omega \times(\tau, T) .
$$


From (33), (34), and the arbitrariness of $\left\{u^{m^{\prime}}\right\}_{m^{\prime} \in \mathbb{N}}$, we have that

$$
G\left(\cdot, u^{m}(\cdot)\right) \rightarrow G(\cdot, u(\cdot)) \text { weakly in } L^{q}\left(\tau, T ; L^{q}(\Omega)\right) .
$$

Then, thanks to the equation satisfied by $\frac{d u^{m}}{d t}$ and the fact that $\operatorname{span}\left\{w_{j}\right\}_{j \geq 1}$ is dense in $V \cap L^{p}(\Omega)$, it is a standard matter to prove that we can pick an element in the equivalence class of $u$ satisfying

$$
(u(t), w)=(u(\tau), w)+\int_{\tau}^{t}\left\langle\Delta u(s)+K\left(s, u_{s}\right)-G(s, u)+F(s), w\right\rangle d s,
$$

for all $t \geq \tau$, for any $w \in V \cap L^{p}(\Omega)$.

To prove that $u(\tau)=u^{\tau}$ we argue similarly to [Anguiano, 2011] and [Anguiano et al., 2010].

Lemma 2. Under the assumptions of Theorem 1, the sequence $u^{m}$ satisfying (30) also satisfies

$$
u^{m} \rightarrow u \text { strongly in } L^{2}\left(\tau, T ; L^{2}\left(\Omega_{n}\right)\right),
$$

for all $T>\tau$ and any $n \geq 1$.

Proof. Let $T>\tau$ and $w \in V_{m}$. Integrating the equality

$$
\frac{d}{d s}\left(u^{m}(s), w\right)=-\left(\nabla u^{m}(s), \nabla w\right)+\left\langle K\left(s, u_{s}^{m}\right)-G\left(s, u^{m}(s)\right)+F(s), w\right\rangle,
$$

between $t$ and $t+a$, with $a \in(0, T-\tau), t \in(\tau, T-a)$, and using the Hölder inequality, we obtain

$$
\begin{aligned}
&\left(u^{m}(t+a)-u^{m}(t), w\right) \leq \int_{t}^{t+a}\left|\nabla u^{m}(s)\right||\nabla w| d s+\int_{t}^{t+a}\left\|K\left(s, u_{s}^{m}\right)\right\|_{-1}|\nabla w| d s \\
& \quad \quad \int_{t}^{t+a}\left|G\left(s, u^{m}(s)\right)\right|_{q}|w|_{p} d s+\int_{t}^{t+a}\|F(s)\|_{-1}|\nabla w| d s \\
& \leq|\nabla w| a^{1 / 2}\left\|u^{m}\right\|_{L^{2}(\tau, T ; V)}+|\nabla w| a^{1 / 2}\left\|K\left(\cdot, u^{m}\right)\right\|_{L^{2}\left(\tau, T ; V^{\prime}\right)} \\
& \quad+|w|_{p} a^{1 / p}\left|G\left(\cdot, u^{m}\right)\right|_{L^{q}\left(\tau, T ; L^{q}(\Omega)\right)}+|\nabla w| a^{1 / 2}\|F\|_{L^{2}\left(\tau, T ; V^{\prime}\right)} .
\end{aligned}
$$

By $(7),(10)$ and $(29)$, we obtain that $G\left(\cdot, u^{m}\right)$ is bounded in $L^{q}\left(\tau, T ; L^{q}(\Omega)\right)$ and $K\left(\cdot, u_{.}^{m}\right)$ is bounded in $L^{2}\left(\tau, T ; V^{\prime}\right)$ and that there exists a constant $C^{(1)}$ (depending on $T$ and $\tau$ ) such that

$$
\left(u^{m}(t+a)-u^{m}(t), w\right) \leq C^{(1)}\left(a^{1 / p}+a^{1 / 2}\right)\left(|\nabla w|+|w|_{p}\right),
$$

for all $w \in V_{m}, m \geq 1, a \in(0, T-\tau), t \in(\tau, T-a)$.

If we take in the last inequality $w=u^{m}(t+a)-u^{m}(t) \in V_{m}$ and integrate between $\tau$ and $T-a$, we obtain

$$
\int_{\tau}^{T-a}\left|u^{m}(t+a)-u^{m}(t)\right|^{2} d t \leq 2 C^{(1)}\left(a^{1 / p}+a^{1 / 2}\right) \int_{\tau}^{T}\left|\nabla u^{m}(s)\right| d s+2 C^{(1)}\left(a^{1 / p}+a^{1 / 2}\right) \int_{\tau}^{T}\left|u^{m}(s)\right|_{p} d s,
$$

and using the Hölder inequality,

$$
\begin{aligned}
\int_{\tau}^{T-a}\left|u^{m}(t+a)-u^{m}(t)\right|^{2} d t \leq & 2 C^{(1)}\left(a^{1 / p}+a^{1 / 2}\right)(T-\tau)^{1 / 2}\left\|u^{m}\right\|_{L^{2}(\tau, T ; V)} \\
& +2 C^{(1)}\left(a^{1 / p}+a^{1 / 2}\right)(T-\tau)^{1 / q}\left\|u^{m}\right\|_{L^{p}\left(\tau, T ; L^{p}(\Omega)\right)} .
\end{aligned}
$$

From (29) we deduce that there exists a constant $C^{(2)}$, depending on $T$ and $\tau$, such that

$$
\int_{\tau}^{T-a}\left|u^{m}(t+a)-u^{m}(t)\right|^{2} d t \leq C^{(2)}\left(a^{1 / p}+a^{1 / 2}\right),
$$


for all $m$, and all $a \in(0, T-\tau)$, and thus

$$
\lim _{a \rightarrow 0}\left(\sup _{m} \int_{\tau}^{T-a}\left|u^{m}(t+a)-u^{m}(t)\right|^{2} d t\right)=0,
$$

for all $T>\tau$.

On the other hand, let $\phi \in C^{1}([0,+\infty))$ be a function such that

$$
0 \leq \phi(s) \leq 1, \quad \phi(s)=1 \quad \forall s \in[0,1], \quad \phi(s)=0 \quad \forall s \geq 2 .
$$

For each $m$ and $n \geq 1$, we define

$$
v^{m, n}(x, t)=\phi\left(\frac{|x|_{\mathbb{R}^{N}}^{2}}{n^{2}}\right) u^{m}(x, t) \quad \forall x \in \Omega_{2 n}, \forall m, \forall n \geq 1 .
$$

We obtain from (29) that, for all $n \geq 1$, the sequence $\left\{v^{m, n}\right\}_{m \geq 1}$ is bounded in $L^{\infty}\left(\tau, T ; L^{2}\left(\Omega_{2 n}\right)\right) \cap$ $L^{p}\left(\tau, T ; L^{p}\left(\Omega_{2 n}\right)\right) \cap L^{2}\left(\tau, T ; H_{0}^{1}\left(\Omega_{2 n}\right)\right)$, for all $T>\tau$.

In particular, it follows that

$$
\int_{\tau}^{\tau+a}\left|v^{m, n}(t)\right|_{L^{2}\left(\Omega_{2 n}\right)}^{2} d t+\int_{T-a}^{T}\left|v^{m, n}(t)\right|_{L^{2}\left(\Omega_{2 n}\right)}^{2} d t \leq 2 a\left\|v^{m, n}\right\|_{L^{\infty}\left(\tau, T ; L^{2}\left(\Omega_{2 n}\right)\right)}^{2},
$$

and therefore

$$
\limsup _{a \rightarrow 0} \sup _{m}\left(\int_{\tau}^{\tau+a}\left|v^{m, n}(t)\right|_{L^{2}\left(\Omega_{2 n}\right)}^{2} d t+\int_{T-a}^{T}\left|v^{m, n}(t)\right|_{L^{2}\left(\Omega_{2 n}\right)}^{2} d t\right)=0 .
$$

From (36) we see that for each $n \geq 1$,

$$
\lim _{a \rightarrow 0}\left(\sup _{m} \int_{\tau}^{T-a}\left|v^{m, n}(t+a)-v^{m, n}(t)\right|_{L^{2}\left(\Omega_{2 n}\right)}^{2} d t\right)=0 .
$$

Moreover, as $\Omega_{2 n}$ is a bounded set, then $H_{0}^{1}\left(\Omega_{2 n}\right)$ is included in $L^{2}\left(\Omega_{2 n}\right)$ with compact injection.

Then, by the compactness Theorem 13.3 and Remark 13.1 of [Temam, 1983] with $X=L^{2}\left(\Omega_{2 n}\right)$, $Y=H_{0}^{1}\left(\Omega_{2 n}\right), r=2$ and $\mathcal{G}=\left\{v^{m, n}\right\}_{m \geq 1}$, we obtain that

$$
\left\{v^{m, n}\right\}_{m \geq 1} \text { is relatively compact in } L^{2}\left(\tau, T ; L^{2}\left(\Omega_{2 n}\right)\right),
$$

and thus, taking into account that $v^{m, n}(x, t)=u^{m}(x, t)$ for all $x \in \Omega_{n}$, we deduce that, in particular, for all $n \geq 1$

$$
\left\{u_{\left.\right|_{\Omega_{n}}}^{m}\right\}_{m \geq 1} \text { is relatively compact in } L^{2}\left(\tau, T ; L^{2}\left(\Omega_{n}\right)\right) \text {. }
$$

By a contradiction argument, it is not difficult to conclude from (30) and (38) that $u^{m} \rightarrow u$ strongly in $L^{2}\left(\tau, T ; L^{2}\left(\Omega_{n}\right)\right)$, for all $n \geq 1$.

\section{Preliminaries on the abstract theory of pullback attractors}

As the uniqueness of the Cauchy problem fails to be true for our equation, we have to work with set-valued non-autonomous dynamical systems.

First we recall some basic definitions for set-valued non-autonomous dynamical systems and establish a sufficient condition for the existence of pullback attractors for these systems. The results in this section can be found in [Anguiano, 2011], [Anguiano et al., 2010, 2012], [Caraballo \& Kloeden, 2009] and [Marín-Rubio \& Real, 2010], among others (see [Melnik \& Valero, 1998] for the autonomous case).

Let $X=\left(X, d_{X}\right)$ be a metric space, let $\mathcal{P}(X)$ denote the family of all nonempty subsets of $X$, and $\mathbb{R}_{d}^{2}:=\left\{(t, s) \in \mathbb{R}^{2}: t \geq s\right\}$. 
Definition 3.1. A multi-valued map $U: \mathbb{R}_{d}^{2} \times X \rightarrow \mathcal{P}(X)$ is called a multi-valued non-autonomous dynamical system (MNDS) on $X$ (also named a multi-valued process on $X$ ) if

$$
\begin{gathered}
U(\tau, \tau, x)=\{x\} \text { for all } \tau \in \mathbb{R}, x \in X, \\
U(t, \tau, x) \subset U(t, s, U(s, \tau, x)) \text { for all } \tau \leq s \leq t, x \in X,
\end{gathered}
$$

where $U(t, \tau, V):=\bigcup_{x_{0} \in V} U\left(t, \tau, x_{0}\right)$ for any non-empty set $V \subset X$.

An MNDS is said to be strict if

$$
U(t, \tau, x)=U(t, s, U(s, \tau, x)) \text { for all } \tau \leq s \leq t, x \in X .
$$

Definition 3.2. An MNDS $U$ on $X$ is said to be upper-semicontinuous if for all $t \geq \tau$ the mapping $U(t, \tau, \cdot)$ is upper-semicontinuous from $X$ into $\mathcal{P}(X)$, i.e., for any $x_{0} \in X$ and for every neighborhood $\mathcal{N}$ in $X$ of the set $U\left(t, \tau, x_{0}\right)$, there exists $\delta>0$ such that $U(t, \tau, y) \subset \mathcal{N}$ whenever $d_{X}\left(x_{0}, y\right)<\delta$.

Let $\mathcal{D}$ be a class of sets parameterized in time, $\widehat{D}=\{D(t): t \in \mathbb{R}\} \subset \mathcal{P}(X)$. The class $\mathcal{D}$ will be called a universe in $\mathcal{P}(X)$. We will say that the class $\mathcal{D}$ is inclusion-closed if $\widehat{D} \in \mathcal{D}$ and $\emptyset \neq D^{\prime}(t) \subset D(t)$ for all $t \in \mathbb{R}$, imply that $\widehat{D}^{\prime}=\left\{D^{\prime}(t): t \in \mathbb{R}\right\}$ belongs to $\mathcal{D}$.

Definition 3.3. We say that a family $\widehat{D}_{0}=\left\{D_{0}(t): t \in \mathbb{R}\right\} \subset \mathcal{P}(X)$ is pullback $\mathcal{D}$-absorbing for the MNDS $U$ if for every $\widehat{D} \in \mathcal{D}$ and every $t \in \mathbb{R}$, there exists $\tau(t, \widehat{D}) \leq t$ such that

$$
U(t, \tau, D(\tau)) \subset D_{0}(t) \text { for all } \tau \leq \tau(t, \widehat{D}) .
$$

Definition 3.4. The MNDS $U$ is pullback asymptotically compact with respect to a family $\widehat{B}=$ $\{B(t): t \in \mathbb{R}\} \subset \mathcal{P}(X)$ (or pullback $\widehat{B}$-asymptotically compact) if for all $t \in \mathbb{R}$ and every sequence $\tau_{n} \leq t$ tending to $-\infty$, any sequence $y_{n} \in U\left(t, \tau_{n}, B\left(\tau_{n}\right)\right)$ is relatively compact in $X$.

We denote by $\operatorname{dist}_{X}\left(\mathcal{O}_{1}, \mathcal{O}_{2}\right)$ the Hausdorff semi-distance in $X$ between two sets $\mathcal{O}_{1}$ and $\mathcal{O}_{2}$, defined as

$$
\operatorname{dist}_{X}\left(\mathcal{O}_{1}, \mathcal{O}_{2}\right)=\sup _{x \in \mathcal{O}_{1}} \inf _{y \in \mathcal{O}_{2}} d_{X}(x, y) \quad \text { for } \mathcal{O}_{1}, \mathcal{O}_{2} \subset X .
$$

Definition 3.5. A family $\mathcal{A}=\{\mathcal{A}(t): t \in \mathbb{R}\} \subset \mathcal{P}(X)$ is said to be a global pullback $\mathcal{D}$-attractor for the MNDS $U$ if it satisfies

(1) $\mathcal{A}(t)$ is compact for any $t \in \mathbb{R}$,

(2) $\mathcal{A}$ is pullback $\mathcal{D}$-attracting, i.e.

$$
\lim _{\tau \rightarrow-\infty} \operatorname{dist}_{X}(U(t, \tau, D(\tau)), \mathcal{A}(t))=0 \quad \forall t \in \mathbb{R},
$$

for all $\widehat{D} \in \mathcal{D}$,

(3) $\mathcal{A}$ is negatively invariant, i.e.,

$$
\mathcal{A}(t) \subset U(t, \tau, \mathcal{A}(\tau)) \text {, for any }(t, \tau) \in \mathbb{R}_{d}^{2} .
$$

$\mathcal{A}$ is said to be a strict global pullback $\mathcal{D}$-attractor if the invariance property in the third item is strict, i.e.,

$$
\mathcal{A}(t)=U(t, \tau, \mathcal{A}(\tau)), \text { for }(t, \tau) \in \mathbb{R}_{d}^{2} .
$$

Theorem 2. Assume that $\widehat{D}_{0}=\left\{D_{0}(t): t \in \mathbb{R}\right\} \subset \mathcal{P}(X)$ is pullback $\mathcal{D}$-absorbing for a MNDS U, which is also pullback $\widehat{D}_{0}$-asymptotically compact. Then, the family $\mathcal{A}_{\mathcal{D}}=\left\{\mathcal{A}_{\mathcal{D}}(t): t \in \mathbb{R}\right\}$ given by

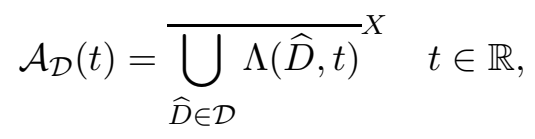

where $\Lambda(\widehat{D}, t)=\bigcap_{s \leq t \tau \leq s} \bar{\bigcup} U(t, \tau, D(\tau))^{X}$, satisfies the following properties: 
(1) For each $t \in \mathbb{R}$ the set $\mathcal{A}_{\mathcal{D}}(t)$ is a nonempty compact subset of $X$, and

$$
\mathcal{A}_{\mathcal{D}}(t) \subset \Lambda\left(\widehat{D}_{0}, t\right) .
$$

(2) $\mathcal{A}_{\mathcal{D}}$ is pullback $\mathcal{D}$-attracting, and in fact is the minimal family of closed sets that attracts pullback to all elements of $\mathcal{D}$.

(3) If $\widehat{D}_{0} \in \mathcal{D}$, then $\mathcal{A}_{\mathcal{D}}(t)=\Lambda\left(\widehat{D}_{0}, t\right) \subset{\overline{D_{0}(t)}}^{X}$, for all $t \in \mathbb{R}$.

(4) If $U$ is upper semi-continuous and with closed values, $\mathcal{A}_{\mathcal{D}}$ is a global pullback $\mathcal{D}$-attractor for $U$.

(5) If $\widehat{D}_{0} \in \mathcal{D}$, each $D_{0}(t)$ is closed and the universe $\mathcal{D}$ is inclusion-closed, then $\mathcal{A}_{\mathcal{D}} \in \mathcal{D}$. If moreover $U$ is upper semi-continuous and with closed values, $\mathcal{A}_{\mathcal{D}}$ is the unique global pullback $\mathcal{D}$-attractor belonging to $\mathcal{D}$. In this case, if moreover $U$ is strict, then $\mathcal{A}_{\mathcal{D}}$ is a strict global pullback $\mathcal{D}$-attractor for $U$.

Proof. See [Anguiano, 2011], [Anguiano et al., 2012], [Caraballo \& Kloeden, 2009] and [Marín-Rubio \& Real, 2010].

\section{Pullback attractors for problem (5)}

In this section we define a multivalued non-autonomous dynamical system generated by the weak solutions of (5) and prove the existence of pullback attractors for it. First, we need a priori estimates and a continuity result which are established in the next subsections.

\subsection{A priori estimates}

Let $S\left(\left(u^{\tau}, \psi\right), \tau\right)$ be the set of all weak solutions $u=u\left(\cdot ; \tau,\left(u^{\tau}, \psi\right)\right)$ of $(5)$ corresponding to initial data $\left(u^{\tau}, \psi\right) \in \mathcal{H}$ and $\tau \in \mathbb{R}$. We define the multivalued map $U: \mathbb{R}_{d} \times \mathcal{H} \rightarrow \mathcal{P}(\mathcal{H})$ as follows

$$
U\left(t, \tau,\left(u^{\tau}, \psi\right)\right)=\left\{\left(u(t), u_{t}\right): u=u\left(\cdot ; \tau,\left(u^{\tau}, \psi\right)\right) \in S\left(\left(u^{\tau}, \psi\right), \tau\right)\right\} \subset \mathcal{H} .
$$

Lemma 3. Under the assumptions of Theorem 1, the multi-valued mapping $U$ defined by (40) is a strict MNDS on $\mathcal{H}$.

Proof. It is easy to check that $U$ satisfies the first part in Definition 3.1.

Let us now fix $\tau \leq t$ and $\left(u^{\tau}, \psi\right) \in \mathcal{H}$. Consider $(\phi, \varphi) \in U\left(t, \tau,\left(u^{\tau}, \psi\right)\right)$. Then, from the definition of $U$, there exists a solution $u \in S\left(\left(u^{\tau}, \psi\right), \tau\right)$ such that $u(t)=\phi$ and $u_{t}=\varphi$. If $\tau \leq s \leq t$, then $\left(u(s), u_{s}\right) \in U\left(s, \tau,\left(u^{\tau}, \psi\right)\right)$, and obviously,

$$
(\phi, \varphi)=\left(u(t), u_{t}\right) \in U\left(t, s,\left(u(s), u_{s}\right)\right) \subset U\left(t, s, U\left(s, \tau,\left(u^{\tau}, \psi\right)\right)\right) .
$$

Thus,

$$
U\left(t, \tau,\left(u^{\tau}, \psi\right)\right) \subset U\left(t, s, U\left(s, \tau,\left(u^{\tau}, \psi\right)\right)\right) \quad \forall \tau \leq s \leq t .
$$

To prove that the MNDS is strict, let us fix $\tau \leq s \leq t$ and $\left(u^{\tau}, \psi\right) \in \mathcal{H}$, and be given $(\phi, \varphi) \in$ $U\left(t, s, U\left(s, \tau,\left(u^{\tau}, \psi\right)\right)\right)$. Then, there exists a solution $y \in S\left(\left(u^{\tau}, \psi\right), \tau\right)$ such that there exists $u \in$ $S\left(\left(y(s), y_{s}\right), s\right)$ satisfying $(\phi, \varphi)=\left(u(t), u_{t}\right)$.

We now define

$$
z(r)=\left\{\begin{array}{cc}
y(r) & \text { if } r \leq s \\
u(r) & \text { if } s \leq r
\end{array}\right.
$$

It can be seen that then

$$
z_{r}=\left\{\begin{array}{cc}
y_{r} & \text { if } r \leq s \\
u_{r} & \text { if } s \leq r
\end{array}\right.
$$

and $z \in S\left(\left(u^{\tau}, \psi\right), \tau\right)$. Consequently,

$$
(\phi, \varphi)=\left(u(t), u_{t}\right)=\left(z(t), z_{t}\right) \in U\left(t, \tau,\left(u^{\tau}, \psi\right)\right) .
$$


This means that

$$
U\left(t, s, U\left(s, \tau,\left(u^{\tau}, \psi\right)\right)\right) \subset U\left(t, \tau,\left(u^{\tau}, \psi\right)\right) \forall \tau \leq s \leq t .
$$

Now, we additionally assume that for the constant $\lambda_{1}$ given in (1), we have

$$
\int_{-\infty}^{t} e^{\lambda_{1} s} c(s) d s<+\infty \quad \forall t \in \mathbb{R}
$$

where the function $c=2 c_{1}+c_{3}$ has been defined in Lemma 1 .

Remark 4.1. Observe that thanks to (41), we have

$$
\lim _{t \rightarrow-\infty} \int_{-\infty}^{t} e^{\lambda_{1} s} c(s) d s=0 .
$$

Let $\mathcal{R}_{\lambda_{1}}$ be the set of all functions $r: \mathbb{R} \rightarrow(0,+\infty)$ such that

$$
\lim _{t \rightarrow-\infty} e^{\lambda_{1} t} r^{2}(t)=0
$$

and denote by $\mathcal{D}_{\lambda_{1}}$ the class of all families $\widehat{D}=\{D(t): t \in \mathbb{R}\} \subset \mathcal{P}(\mathcal{H})$ such that $D(t) \subset \bar{B}_{\mathcal{H}}\left(0, r_{\widehat{D}}(t)\right)$ for some $r_{\widehat{D}} \in \mathcal{R}_{\lambda_{1}}$, where $\bar{B}_{\mathcal{H}}\left(0, r_{\widehat{D}}(t)\right)$ denotes the closed ball in $\mathcal{H}$ centered at zero with radius $r_{\widehat{D}}(t)$. Observe that the class $\mathcal{D}_{\lambda_{1}}$ is inclusion-closed.

Define

$$
R_{\lambda_{1}}^{2}(t)=1+\left(1+\frac{2}{1-d}\right) e^{-\lambda_{1} t} \int_{-\infty}^{t} e^{\lambda_{1} s} c(s) d s+\left(4+\frac{8}{1-d}\right) e^{-\lambda_{1} t} \int_{-\infty}^{t} e^{\lambda_{1} s}\|F(s)\|_{-1}^{2} d s .
$$

We have the existence of a pullback absorbing family for $U$.

Lemma 4. Assume that the assumptions (6), (7), (H1)-(H3), (H6) and (41) are satisfied. Then the balls $B_{\lambda_{1}}=\bar{B}_{\mathcal{H}}\left(0, R_{\lambda_{1}}(t)\right)$, where $R_{\lambda_{1}}(t)>0$ is given by (43) for each $t \in \mathbb{R}$, form a family $\widehat{B}_{\lambda_{1}} \in \mathcal{D}_{\lambda_{1}}$ which is pullback $\mathcal{D}_{\lambda_{1}}$-absorbing for the MNDS U defined by (40).

Proof. The fact that the family $\widehat{B}_{\lambda_{1}}$ is pullback $\mathcal{D}_{\lambda_{1}}$-absorbing for the MNDS $U$ is an immediate consequence of Lemma 1.

On the other hand, by conditions (15) and (42), it is evident that $\widehat{B}_{\lambda_{1}} \in \mathcal{D}_{\lambda_{1}}$.

Lemma 5. Assume that $G$ is given by (16), with $g:(x, t) \in \Omega \times \mathbb{R} \mapsto g(x, t, r) \in \mathbb{R}$ measurable for all $r \in \mathbb{R}, g(x, t, \cdot) \in C(\mathbb{R})$ for a.e. $(x, t) \in \Omega \times \mathbb{R}$, satisfying (3) and (4). Suppose also that assumptions (H1), (H3), (H5) and (H6) are satisfied, and that (41) holds, with $c_{1}(\cdot)=\int_{\Omega} \delta_{1}(x, \cdot) d x$. Then, for any real numbers $t_{1} \leq t_{2}$ and any $\varepsilon>0$, there exist $T=T\left(t_{1}, t_{2}, \varepsilon, \widehat{B}_{\lambda_{1}}\right) \leq t_{1}$ and $M=M\left(t_{1}, t_{2}, \varepsilon, \widehat{B}_{\lambda_{1}}\right) \geq 1$ such that for any $\left(u^{\tau}, \psi\right) \in B_{\lambda_{1}}(\tau)$ and any weak solution $u \in S\left(\left(u^{\tau}, \psi\right), \tau\right)$,

$$
\int_{\Omega \cap\left\{|x|_{\left.\mathbb{R}^{N} \geq 2 n\right\}}\right.} u^{2}(x, t) d x \leq \varepsilon, \quad \forall \tau \leq T, \quad t \in\left[t_{1}, t_{2}\right], \quad n \geq M .
$$

Proof. Let $\tau \in \mathbb{R},\left(u^{\tau}, \psi\right) \in \mathcal{H}$ and $u \in S\left(\left(u^{\tau}, \psi\right), \tau\right)$ be fixed. Take a smooth function $\theta \in C^{1}([0,+\infty))$ satisfying

$$
0 \leq \theta(s) \leq 1, \quad \theta(s)=0 \quad \forall s \in[0,1], \quad \theta(s)=1 \quad \forall s \geq 2 .
$$

The function $|\theta u(t)|^{2}=\int_{\Omega} \theta^{2}\left(\frac{|x|^{2}}{n^{2}}\right)|u(x, t)|^{2} d x$ is absolutely continuous and $\frac{d}{d t}|\theta u|^{2}=2\left\langle\frac{d u}{d t}, \theta^{2} u\right\rangle$ for a.e. $t$. On the other hand, we observe that

$$
\partial_{i}\left(\theta^{2}\left(\frac{|x|_{\mathbb{R}^{N}}^{2}}{n^{2}}\right) u(x, t)\right)=\theta^{2}\left(\frac{|x|_{\mathbb{R}^{N}}^{2}}{n^{2}}\right) \partial_{i} u(x, t)+\frac{4 x_{i}}{n^{2}} \theta\left(\frac{|x|_{\mathbb{R}^{N}}^{2}}{n^{2}}\right) \theta^{\prime}\left(\frac{|x|_{\mathbb{R}^{N}}^{2}}{n^{2}}\right) u(x, t) .
$$


Hence, taking into account (H5), (H6) and Remark 2.2, we obtain for every $t \geq \tau$,

$$
\begin{aligned}
& \frac{1}{2} \frac{d}{d t} \int_{\Omega} \theta^{2}\left(\frac{|x|_{\mathbb{R}^{N}}^{2}}{n^{2}}\right) u^{2}(x, t) d x+\int_{\Omega} \theta^{2}\left(\frac{|x|_{\mathbb{R}^{N}}^{2}}{n^{2}}\right)|\nabla u(x, t)|^{2} d x \\
&+\frac{4}{n^{2}} \int_{\Omega} \theta^{\prime}\left(\frac{|x|_{\mathbb{R}^{N}}^{2}}{n^{2}}\right) \theta\left(\frac{|x|_{\mathbb{R}^{N}}^{2}}{n^{2}}\right) u(x, t) x \cdot \nabla u(x, t) d x \\
&=-\sum_{i=1}^{N} \int_{\Omega} \theta^{2}\left(\frac{|x|_{\mathbb{R}^{N}}^{2}}{n^{2}}\right) K_{i}\left(t, u_{t}\right) \partial_{i} u(x, t) d x \\
&-\sum_{i=1}^{N} \int_{\Omega} \frac{4 x_{i}}{n^{2}} \theta^{\prime}\left(\frac{|x|_{\mathbb{R}^{N}}^{2}}{n^{2}}\right) \theta\left(\frac{|x|_{\mathbb{R}^{N}}^{2}}{n^{2}}\right) u(x, t) K_{i}\left(t, u_{t}\right) d x \\
&-\int_{\Omega} \theta^{2}\left(\frac{|x|_{\mathbb{R}^{N}}^{2}}{n^{2}}\right) G(t, u) u(x, t) d x-\sum_{i=1}^{N} \int_{\Omega} \theta^{2}\left(\frac{|x|_{\mathbb{R}^{N}}^{2}}{n^{2}}\right) F_{i}(x, t) \partial_{i} u(x, t) d x \\
&-\sum_{i=1}^{N} \int_{\Omega} \frac{4 x_{i}}{n^{2}} \theta^{\prime}\left(\frac{|x|_{\mathbb{R}^{N}}^{2}}{n^{2}}\right) \theta\left(\frac{|x|_{\mathbb{R}^{N}}^{2}}{n^{2}}\right) u(x, t) F_{i}(x, t) d x \\
&= I_{1}+I_{2}+I_{3}+I_{4}+I_{5} .
\end{aligned}
$$

Using the Cauchy-Schwarz inequality, we obtain

$$
I_{1} \leq \frac{1}{8} \int_{\Omega} \theta^{2}\left(\frac{|x|_{\mathbb{R}^{N}}^{2}}{n^{2}}\right)|\nabla u(x, t)|^{2} d x+2 \sum_{i=1}^{N} \int_{\Omega} \theta^{2}\left(\frac{|x|_{\mathbb{R}^{N}}^{2}}{n^{2}}\right) K_{i}^{2}\left(t, u_{t}\right) d x
$$

and

$$
I_{4} \leq \frac{1}{8} \int_{\Omega} \theta^{2}\left(\frac{|x|_{\mathbb{R}^{N}}^{2}}{n^{2}}\right)|\nabla u(x, t)|^{2} d x+2 \sum_{i=1}^{N} \int_{\Omega} \theta^{2}\left(\frac{|x|_{\mathbb{R}^{N}}^{2}}{n^{2}}\right) F_{i}^{2}(x, t) d x .
$$

Using the fact that $\theta^{\prime}\left(\frac{|x|_{\mathbb{R}^{N}}^{2}}{n^{2}}\right)=0$ if $|x|_{\mathbb{R}^{N}}>\sqrt{2} n, \theta^{\prime}\left(\frac{|x|_{\mathbb{R}^{N}}^{2}}{n^{2}}\right) \leq C_{\theta^{\prime}}$ for all $|x|_{\mathbb{R}^{N}} \leq 2 n$, and once more the Cauchy-Schwarz inequality, we obtain

$$
\begin{aligned}
\left|I_{2}\right| & \leq \frac{8}{n} C_{\theta^{\prime}} \sum_{i=1}^{N} \int_{\Omega} \theta\left(\frac{|x|_{\mathbb{R}^{N}}^{2}}{n^{2}}\right)|u(x, t)|\left|K_{i}\left(t, u_{t}\right)\right| d x \\
& \leq \frac{8}{n} C_{\theta^{\prime}} N^{1 / 2}\left(\sum_{i=1}^{N} \int_{\Omega} \theta^{2}\left(\frac{|x|_{\mathbb{R}^{N}}^{2}}{n^{2}}\right) K_{i}^{2}\left(t, u_{t}\right) d x\right)^{1 / 2}\left(\int_{\Omega} u^{2}(x, t) d x\right)^{1 / 2} \\
& \leq \frac{8}{n^{2}} C_{\theta^{\prime}}^{2} N \int_{\Omega} u^{2}(x, t) d x+2 \sum_{i=1}^{N} \int_{\Omega} \theta^{2}\left(\frac{|x|_{\mathbb{R}^{N}}^{2}}{n^{2}}\right) K_{i}^{2}\left(t, u_{t}\right) d x
\end{aligned}
$$

and

$$
\left|I_{5}\right| \leq \frac{8}{n^{2}} C_{\theta^{\prime}}^{2} N \int_{\Omega} u^{2}(x, t) d x+2 \sum_{i=1}^{N} \int_{\Omega} \theta^{2}\left(\frac{|x|_{\mathbb{R}^{N}}^{2}}{n^{2}}\right) F_{i}^{2}(x, t) d x .
$$


From (16) and (3), it follows

$$
\begin{aligned}
I_{3} & =-\int_{\Omega} \theta^{2}\left(\frac{|x|_{\mathbb{R}^{N}}^{2}}{n^{2}}\right) g(x, t, u(x, t)) u(x, t) d x \\
& \leq-\eta \int_{\Omega} \theta^{2}\left(\frac{|x|_{\mathbb{R}^{N}}^{2}}{n^{2}}\right)|u(x, t)|^{p} d x+\int_{\Omega} \theta^{2}\left(\frac{|x|_{\mathbb{R}^{N}}^{2}}{n^{2}}\right) \delta_{1}(x, t) d x \\
& \leq \int_{\Omega} \theta^{2}\left(\frac{|x|_{\mathbb{R}^{N}}^{2}}{n^{2}}\right) \delta_{1}(x, t) d x .
\end{aligned}
$$

Moreover, we have

$$
\begin{aligned}
& \left|\frac{4}{n^{2}} \int_{\Omega} \theta^{\prime}\left(\frac{|x|_{\mathbb{R}^{N}}^{2}}{n^{2}}\right) \theta\left(\frac{|x|_{\mathbb{R}^{N}}^{2}}{n^{2}}\right) u(x, t) x \cdot \nabla u(x, t) d x\right| \\
& \quad \leq \frac{4}{n} C_{\theta^{\prime}} \int_{\Omega} u^{2}(x, t) d x+\frac{4}{n} C_{\theta^{\prime}} \int_{\Omega} \theta^{2}\left(\frac{|x|_{\mathbb{R}^{N}}^{2}}{n^{2}}\right)|\nabla u(x, t)|^{2} d x .
\end{aligned}
$$

From (45)-(51) we deduce

$$
\begin{gathered}
\frac{1}{2} \frac{d}{d t} \int_{\Omega} \theta^{2}\left(\frac{|x|_{\mathbb{R}^{N}}^{2}}{n^{2}}\right) u^{2}(x, t) d x+\left(\frac{3}{4}-\frac{4}{n} C_{\theta^{\prime}}\right) \int_{\Omega} \theta^{2}\left(\frac{|x|_{\mathbb{R}^{N}}^{2}}{n^{2}}\right)|\nabla u(x, t)|^{2} d x \\
\leq \int_{\Omega} \theta^{2}\left(\frac{|x|_{\mathbb{R}^{N}}^{2}}{n^{2}}\right) \delta_{1}(x, t) d x+\frac{4}{n} C_{\theta^{\prime}} \int_{\Omega} u^{2}(x, t) d x+\frac{16}{n^{2}} C_{\theta^{\prime}}^{2} N \int_{\Omega} u^{2}(x, t) d x \\
\quad+4 \sum_{i=1}^{N} \int_{\Omega} \theta^{2}\left(\frac{|x|_{\mathbb{R}^{N}}^{2}}{n^{2}}\right) K_{i}^{2}\left(t, u_{t}\right) d x+4 \sum_{i=1}^{N} \int_{\Omega} \theta^{2}\left(\frac{|x|_{\mathbb{R}^{N}}^{2}}{n^{2}}\right) F_{i}^{2}(x, t) d x
\end{gathered}
$$

On the other hand,

$$
\begin{aligned}
&\left|\nabla\left(\theta\left(\frac{|x|_{\mathbb{R}^{N}}^{2}}{n^{2}}\right) u(x, t)\right)\right|^{2}= \theta^{2} \\
&\left(\frac{|x|_{\mathbb{R}^{N}}^{2}}{n^{2}}\right)|\nabla u(x, t)|^{2}+\frac{4|x|_{\mathbb{R}^{N}}^{2}}{n^{4}}\left(\theta^{\prime}\left(\frac{|x|_{\mathbb{R}^{N}}^{2}}{n^{2}}\right)\right)^{2} u^{2}(x, t) \\
&+\frac{4}{n^{2}} \theta^{\prime}\left(\frac{|x|_{\mathbb{R}^{N}}^{2}}{n^{2}}\right) u(x, t) \theta\left(\frac{|x|_{\mathbb{R}^{N}}^{2}}{n^{2}}\right) x \cdot \nabla u(x, t),
\end{aligned}
$$

and therefore

$$
\begin{aligned}
\int_{\Omega}\left|\nabla\left(\theta\left(\frac{|x|_{\mathbb{R}^{N}}^{2}}{n^{2}}\right) u(x, t)\right)\right|^{2} d x \leq & \left(1+\frac{4}{n} C_{\theta^{\prime}}\right) \int_{\Omega} \theta^{2}\left(\frac{|x|_{\mathbb{R}^{N}}^{2}}{n^{2}}\right)|\nabla u(x, t)|^{2} d x \\
& +\left(\frac{8}{n^{2}} C_{\theta^{\prime}}^{2}+\frac{4}{n} C_{\theta^{\prime}}\right) \int_{\Omega} u^{2}(x, t) d x
\end{aligned}
$$

From this inequality and (1) we obtain

$$
\begin{aligned}
\int_{\Omega} \theta^{2}\left(\frac{|x|_{\mathbb{R}^{N}}^{2}}{n^{2}}\right)|\nabla u(x, t)|^{2} d x \geq & \left(\frac{n}{n+4 C_{\theta^{\prime}}}\right) \lambda_{1} \int_{\Omega} \theta^{2}\left(\frac{|x|_{\mathbb{R}^{N}}^{2}}{n^{2}}\right) u^{2}(x, t) d x \\
& -\left(\frac{n}{n+4 C_{\theta^{\prime}}}\right)\left(\frac{8}{n^{2}} C_{\theta^{\prime}}^{2}+\frac{4}{n} C_{\theta^{\prime}}\right) \int_{\Omega} u^{2}(x, t) d x .
\end{aligned}
$$


Assume now that $\frac{3}{4}-\frac{4}{n} C_{\theta^{\prime}}>0$ (which holds true for $n$ large enough). Then, from (52) and (53), we have

$$
\begin{aligned}
\frac{1}{2} \frac{d}{d t} \int_{\Omega} \theta^{2}\left(\frac{|x|_{\mathbb{R}^{N}}^{2}}{n^{2}}\right) u^{2}(x, t) d x+\left(\frac{3}{4}-\frac{4}{n} C_{\theta^{\prime}}\right)\left(\frac{n}{n+4 C_{\theta^{\prime}}}\right) \lambda_{1} \int_{\Omega} \theta^{2}\left(\frac{|x|_{\mathbb{R}^{N}}^{2}}{n^{2}}\right) u^{2}(x, t) d x \\
\leq\left(\frac{4 C_{\theta^{\prime}}}{n}+\frac{16 C_{\theta^{\prime}}^{2} N}{n^{2}}+\left(\frac{n}{n+4 C_{\theta^{\prime}}}\right)\left(\frac{8 C_{\theta^{\prime}}^{2}}{n^{2}}+\frac{4 C_{\theta^{\prime}}}{n}\right)\left(\frac{3}{4}-\frac{4 C_{\theta^{\prime}}}{n}\right)\right) \int_{\Omega} u^{2}(x, t) d x \\
\quad+\int_{\Omega} \theta^{2}\left(\frac{|x|_{\mathbb{R}^{N}}^{2}}{n^{2}}\right) \delta_{1}(x, t) d x+4 \sum_{i=1}^{N} \int_{\Omega} \theta^{2}\left(\frac{|x|_{\mathbb{R}^{N}}^{2}}{n^{2}}\right) K_{i}^{2}\left(t, u_{t}\right) d x \\
+4 \sum_{i=1}^{N} \int_{\Omega} \theta^{2}\left(\frac{|x|_{\mathbb{R}^{N}}^{2}}{n^{2}}\right) F_{i}^{2}(x, t) d x .
\end{aligned}
$$

Evidently, there exists $n_{0}$ such that for all $n \geq n_{0}$ we have

$$
\left(\frac{3}{4}-\frac{4}{n} C_{\theta^{\prime}}\right)\left(\frac{n}{n+4 C_{\theta^{\prime}}}\right)>\frac{1}{2}
$$

Then, from (54) we obtain

$$
\begin{aligned}
& \frac{d}{d t} \int_{\Omega} \theta^{2}\left(\frac{|x|_{\mathbb{R}^{N}}^{2}}{n^{2}}\right) u^{2}(x, t) d x+\lambda_{1} \int_{\Omega} \theta^{2}\left(\frac{|x|_{\mathbb{R}^{N}}^{2}}{n^{2}}\right) u^{2}(x, t) d x \\
& \leq 2\left(\frac{4 C_{\theta^{\prime}}}{n}+\frac{16 C_{\theta^{\prime}}^{2} N}{n^{2}}+\left(\frac{n}{n+4 C_{\theta^{\prime}}}\right)\left(\frac{8 C_{\theta^{\prime}}^{2}}{n^{2}}+\frac{4 C_{\theta^{\prime}}}{n}\right)\left(\frac{3}{4}-\frac{4 C_{\theta^{\prime}}}{n}\right)\right) \int_{\Omega} u^{2}(x, t) d x \\
& \quad+2 \int_{\Omega} \theta^{2}\left(\frac{|x|_{\mathbb{R}^{N}}^{2}}{n^{2}}\right) \delta_{1}(x, t) d x+8 \sum_{i=1}^{N} \int_{\Omega} \theta^{2}\left(\frac{|x|_{\mathbb{R}^{N}}^{2}}{n^{2}}\right) K_{i}^{2}\left(t, u_{t}\right) d x \\
& \quad+8 \sum_{i=1}^{N} \int_{\Omega} \theta^{2}\left(\frac{|x|_{\mathbb{R}^{N}}^{2}}{n^{2}}\right) F_{i}^{2}(x, t) d x, \quad \text { for all } n \geq n_{0} .
\end{aligned}
$$

We observe that

$$
\begin{aligned}
& \frac{4 C_{\theta^{\prime}}}{n}+\frac{16 C_{\theta^{\prime}}^{2} N}{n^{2}}+\left(\frac{n}{n+4 C_{\theta^{\prime}}}\right)\left(\frac{8 C_{\theta^{\prime}}^{2}}{n^{2}}+\frac{4 C_{\theta^{\prime}}}{n}\right)\left(\frac{3}{4}-\frac{4 C_{\theta^{\prime}}}{n}\right) \\
& \leq \frac{1}{n}\left(7 C_{\theta^{\prime}}+22 C_{\theta^{\prime}}^{2} N\right) .
\end{aligned}
$$

Thus, if we denote $\widehat{C}=14 C_{\theta^{\prime}}+44 C_{\theta^{\prime}}^{2} N$, from (55) we obtain

$$
\begin{aligned}
\frac{d}{d t}\left(e^{\lambda_{1} t} \int_{\Omega} \theta^{2}\left(\frac{|x|_{\mathbb{R}^{N}}^{2}}{n^{2}}\right) u^{2}(x, t) d x\right) \leq & \frac{\widehat{C}}{n} e^{\lambda_{1} t} \int_{\Omega} u^{2}(x, t) d x+2 e^{\lambda_{1} t} \int_{\Omega} \theta^{2}\left(\frac{|x|_{\mathbb{R}^{N}}^{2}}{n^{2}}\right) \delta_{1}(x, t) d x \\
& +8 \sum_{i=1}^{N} e^{\lambda_{1} t} \int_{\Omega} \theta^{2}\left(\frac{|x|_{\mathbb{R}^{N}}^{2}}{n^{2}}\right) K_{i}^{2}(t, u t) d x \\
& +8 \sum_{i=1}^{N} e^{\lambda_{1} t} \int_{\Omega} \theta^{2}\left(\frac{|x|_{\mathbb{R}^{N}}^{2}}{n^{2}}\right) F_{i}^{2}(x, t) d x
\end{aligned}
$$


Integrating now between $\tau$ and $t$, and using the properties of $\theta$, we have

$$
\begin{aligned}
& \int_{\Omega \cap\left\{|x|_{\left.\mathbb{R}^{N} \geq 2 n\right\}} u^{2}(x, t) d x \leq\right.} e^{-\lambda_{1} t} e^{\lambda_{1} \tau} \int_{\Omega} \theta^{2}\left(\frac{|x|_{\mathbb{R}^{N}}^{2}}{n^{2}}\right)\left(u^{\tau}(x)\right)^{2} d x+\frac{\widehat{C}}{n} e^{-\lambda_{1} t} \int_{\tau}^{t} e^{\lambda_{1} s}|u(s)|^{2} d s \\
&+8 \sum_{i=1}^{N} e^{-\lambda_{1} t} \int_{-\infty}^{t} e^{\lambda_{1} s} \int_{\Omega} \theta^{2}\left(\frac{|x|_{\mathbb{R}^{N}}^{2}}{n^{2}}\right) K_{i}^{2}\left(s, u_{s}\right) d x d s \\
&+8 \sum_{i=1}^{N} e^{-\lambda_{1} t} \int_{-\infty}^{t} e^{\lambda_{1} s} \int_{\Omega} \theta^{2}\left(\frac{|x|_{\mathbb{R}^{N}}^{2}}{n^{2}}\right) F_{i}^{2}(x, s) d x d s \\
&+2 e^{-\lambda_{1} t} \int_{-\infty}^{t} e^{\lambda_{1} s} \int_{\Omega} \theta^{2}\left(\frac{|x|_{\mathbb{R}^{N}}^{2}}{n^{2}}\right) \delta_{1}(x, s) d x d s,
\end{aligned}
$$

for all $n \geq n_{0}, t \geq \tau$.

On the other hand, from (24), multiplying by $e^{\lambda_{1} t}$, in particular, we obtain

$$
\frac{d}{d t}\left(e^{\lambda_{1} t}|u(t)|^{2}\right)+\frac{e^{\lambda_{1} t}}{2}|\nabla u(t)|^{2} \leq 4 e^{\lambda_{1} t}\left\|K\left(t, u_{t}\right)\right\|_{-1}^{2}+2 e^{\lambda_{1} t} c_{1}(t)+4 e^{\lambda_{1} t}\|F(t)\|_{-1}^{2} .
$$

Using (1) and integrating between $\tau$ and $t$, we have

$$
\begin{aligned}
\frac{\lambda_{1}}{2} \int_{\tau}^{t} e^{\lambda_{1} s}|u(s)|^{2} d s \leq e^{\lambda_{1} \tau}\left|u^{\tau}\right|^{2}+4 \sum_{i=1}^{N} \int_{\tau}^{t} e^{\lambda_{1} s}\left|K_{i}\left(s, u_{s}\right)\right|^{2} d s \\
\quad+2 \int_{\tau}^{t} e^{\lambda_{1} s} c_{1}(s) d s+4 \sum_{i=1}^{N} \int_{\tau}^{t} e^{\lambda_{1} s}\left|F_{i}(s)\right|^{2} d s .
\end{aligned}
$$

Assuming now that $\left(u^{\tau}, \psi\right) \in B_{\lambda_{1}}(\tau)$, we obtain

$$
\begin{aligned}
\int_{\tau}^{t} e^{\lambda_{1} s}|u(s)|^{2} d s \leq & 2 \lambda_{1}^{-1} e^{\lambda_{1} \tau} R_{\lambda_{1}}^{2}(\tau)+8 \lambda_{1}^{-1} \sum_{i=1}^{N} \int_{-\infty}^{t} e^{\lambda_{1} s}\left|K_{i}\left(s, u_{s}\right)\right|^{2} d s \\
& +4 \lambda_{1}^{-1} \int_{-\infty}^{t} e^{\lambda_{1} s} c_{1}(s) d s+8 \lambda_{1}^{-1} \sum_{i=1}^{N} \int_{-\infty}^{t} e^{\lambda_{1} s}\left|F_{i}(s)\right|^{2} d s
\end{aligned}
$$

for all $t \geq \tau$. Let us fix $t_{1} \leq t_{2} \in \mathbb{R}$. Observing that $\lim _{\tau \rightarrow-\infty} e^{\lambda_{1} \tau} R_{\lambda_{1}}^{2}(\tau)=0$, from (14), (15), (41), and (58), we deduce that there exists a constant $C\left(t_{1}, t_{2}\right)$ such that

$$
e^{-\lambda_{1} t} \int_{\tau}^{t} e^{\lambda_{1} s}|u(s)|^{2} d s \leq C\left(t_{1}, t_{2}\right) \quad \forall t \in\left[t_{1}, t_{2}\right], \tau \leq t_{1},
$$

and taking into account (56) and the fact that $0 \leq \theta \leq 1$, we can deduce

$$
\begin{aligned}
\int_{\Omega \cap\left\{|x|_{\left.\mathbb{R}^{N} \geq 2 n\right\}} u^{2}(x, t) d x \leq\right.} e^{-\lambda_{1} t} e^{\lambda_{1} \tau} R_{\lambda_{1}}^{2}(\tau)+\frac{\widehat{C}}{n} C\left(t_{1}, t_{2}\right) & \\
& +8 \sum_{i=1}^{N} e^{-\lambda_{1} t} \int_{-\infty}^{t} e^{\lambda_{1} s} \int_{\Omega} \theta^{2}\left(\frac{|x|_{\mathbb{R}^{N}}^{2}}{n^{2}}\right) K_{i}^{2}\left(s, u_{s}\right) d x d s \\
& +8 \sum_{i=1}^{N} e^{-\lambda_{1} t} \int_{-\infty}^{t} e^{\lambda_{1} s} \int_{\Omega} \theta^{2}\left(\frac{|x|_{\mathbb{R}^{N}}^{2}}{n^{2}}\right) F_{i}^{2}(x, s) d x d s \\
& +2 e^{-\lambda_{1} t} \int_{-\infty}^{t} e^{\lambda_{1} s} \int_{\Omega} \theta^{2}\left(\frac{|x|_{\mathbb{R}^{N}}^{2}}{n^{2}}\right) \delta_{1}(x, s) d x d s,
\end{aligned}
$$

for $n \geq n_{0}$ and $t \in\left[t_{1}, t_{2}\right]$, for every $u \in S\left(\left(u^{\tau}, \psi\right), \tau\right)$, where $\tau \leq t_{1}$ and $\left(u^{\tau}, \psi\right) \in B_{\lambda_{1}}(\tau)$. 
On the other hand, thanks to (14), we have that for every $t \in \mathbb{R}$,

$$
e^{\lambda_{1} s} K_{i}^{2}\left(s, u_{s}\right) \in L^{1}(\Omega \times(-\infty, t)) .
$$

Thus, by the Lebesgue's Dominated Convergence Theorem, we have that for every $t \in\left[t_{1}, t_{2}\right]$,

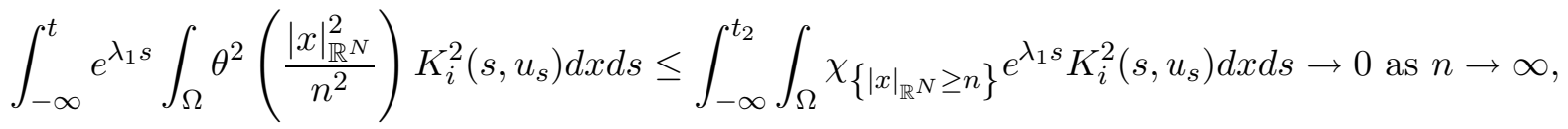

for all $i=1, \ldots, N$.

Analogously, thanks to (15) and (41) we have

$$
\int_{-\infty}^{t} e^{\lambda_{1} s} \int_{\Omega} \theta^{2}\left(\frac{|x|_{\mathbb{R}^{N}}^{2}}{n^{2}}\right) F_{i}^{2}(x, s) d x d s \leq \int_{-\infty}^{t_{2}} \int_{\Omega} \chi_{\left\{|x|_{\mathbb{R}^{N}} \geq n\right\}} e^{\lambda_{1} s} F_{i}^{2}(x, s) d x d s \rightarrow 0 \text { as } n \rightarrow \infty
$$

and

$$
\int_{-\infty}^{t} e^{\lambda_{1} s} \int_{\Omega} \theta^{2}\left(\frac{|x|_{\mathbb{R}^{N}}^{2}}{n^{2}}\right) \delta_{1}(x, s) d x d s \leq \int_{-\infty}^{t_{2}} \int_{\Omega} \chi_{\left\{|x|_{\mathbb{R}^{N}} \geq n\right\}} e^{\lambda_{1} s} \delta_{1}(x, s) d x d s \rightarrow 0 \text { as } n \rightarrow \infty,
$$

for all $i=1, . ., N$ and $t \in\left[t_{1}, t_{2}\right]$.

From (59), (60), (61) and (62) we deduce our lemma.

Lemma 6. Under the assumptions in Lemma 5 , let $\mathcal{K}$ be a relatively compact set in $\mathcal{H}$. Then, for all $\tau \leq T$ and $\varepsilon>0$ there exists $M=M(\tau, T, \varepsilon, \mathcal{K})$ such that

$$
\int_{\Omega \cap\left\{|x|_{\left.\mathbb{R}^{N} \geq 2 n\right\}}\right.} u^{2}(x, t) d x \leq \varepsilon, \forall t \in[\tau, T], \quad \forall n \geq M,
$$

for any $u \in S\left(\left(u^{\tau}, \psi\right), \tau\right)$, where $\left(u^{\tau}, \psi\right) \in \mathcal{K}$ is arbitrary.

Proof. We observe that, as shown in Lemma 5, we have (56) for $n \geq n_{0}$, and $u \in S\left(\left(u^{\tau}, \psi\right), \tau\right)$, where $t \geq \tau$ and $\left(u^{\tau}, \psi\right) \in \mathcal{H}$ are arbitrary.

On the other hand, as $\mathcal{K}$ is a bounded subset of $\mathcal{H}$, from (57) we deduce that for some constant $k_{1}>0$,

$$
\begin{aligned}
\int_{\tau}^{t} e^{\lambda_{1} s}|u(s)|^{2} d s \leq & 2 \lambda_{1}^{-1} e^{\lambda_{1} \tau} k_{1}^{2}+8 \lambda_{1}^{-1} \sum_{i=1}^{N} \int_{-\infty}^{t} e^{\lambda_{1} s}\left|K_{i}\left(s, u_{s}\right)\right|^{2} d s \\
& +4 \lambda_{1}^{-1} \int_{-\infty}^{t} e^{\lambda_{1} s} c_{1}(s) d s+8 \lambda_{1}^{-1} \sum_{i=1}^{N} \int_{-\infty}^{t} e^{\lambda_{1} s}\left|F_{i}(s)\right|^{2} d s
\end{aligned}
$$

and thus there exists a constant $C(\tau, T)$ such that

$$
e^{-\lambda_{1} t} \int_{\tau}^{t} e^{\lambda_{1} s}|u(s)|^{2} d s \leq C(\tau, T) \quad \forall t \in[\tau, T],
$$

for any $u \in S\left(\left(u^{\tau}, \psi\right), \tau\right)$, where $\left(u^{\tau}, \psi\right) \in \mathcal{K}$ is arbitrary.

Finally, as $\mathcal{K}$ is a relatively compact subset of $\mathcal{H}$, then for all $\varepsilon>0$ there exists $n_{\varepsilon} \geq n_{0}$ such that

$$
\int_{\Omega} \theta^{2}\left(\frac{|x|_{\mathbb{R}^{N}}^{2}}{n^{2}}\right)\left(u^{\tau}(x)\right)^{2} d x<\varepsilon \quad \forall\left(u^{\tau}, \psi\right) \in \mathcal{K}, \forall n \geq n_{\varepsilon} .
$$

Otherwise, there would exist an $\varepsilon>0$ and a sequence $\left\{\left(u^{m}, \psi^{m}\right)\right\} \subset \mathcal{K}$ such that

$$
\int_{\Omega} \theta^{2}\left(\frac{|x|_{\mathbb{R}^{N}}^{2}}{m^{2}}\right)\left(u^{m}(x)\right)^{2} d x \geq \varepsilon, \forall m \geq 1
$$


But then, as $\left\{\left(u^{m}, \psi^{m}\right)\right\} \subset \mathcal{K}$, there would exist a convergent subsequence $\left\{u^{\mu}\right\} \subset\left\{u^{m}\right\}$, with $u^{\mu} \rightarrow v$ strongly in $H$ as $\mu \rightarrow \infty$. And thus we would have

$$
\begin{aligned}
\varepsilon & \leq \int_{\Omega} \theta^{2}\left(\frac{|x|_{\mathbb{R}^{N}}^{2}}{\mu^{2}}\right)\left(u^{\mu}(x)\right)^{2} d x \\
& \leq 2 \int_{\Omega} \theta^{2}\left(\frac{|x|_{\mathbb{R}^{N}}^{2}}{\mu^{2}}\right)\left(u^{\mu}(x)-v(x)\right)^{2} d x+2 \int_{\Omega} \theta^{2}\left(\frac{|x|_{\mathbb{R}^{N}}^{2}}{\mu^{2}}\right) v^{2}(x) d x \\
& \leq 2 \int_{\Omega}\left(u^{\mu}(x)-v(x)\right)^{2} d x+2 \int_{\Omega} \theta^{2}\left(\frac{|x|_{\mathbb{R}^{N}}^{2}}{\mu^{2}}\right) v^{2}(x) d x,
\end{aligned}
$$

and therefore, making $\mu \rightarrow \infty$, we would have $\varepsilon \leq 0$, which is a contradiction.

From (56), (63), (64), and taking into account (60)-(62), we deduce our Lemma.

\subsection{A continuity result}

Now, we obtain a continuity result leading to the upper semicontinuity of the MNDS $U$ defined by (40).

Lemma 7. Assume that the assumptions in Lemma 5 are satisfied. Let $\tau \in \mathbb{R}$, and a sequence $\left\{\left(u^{\tau, m}, \psi^{m}\right)\right\} \subset \mathcal{H}$ be given. For each $m \geq 1$ let us fix $u^{m} \in S\left(\left(u^{\tau, m}, \psi^{m}\right), \tau\right)$. Then:

(i) If $\left\{\left(u^{\tau, m}, \psi^{m}\right)\right\}$ converges weakly in $\mathcal{H}$ to an element $\left(u^{\tau}, \psi\right)$, there exists a subsequence $\left\{u^{\mu}\right\} \subset\left\{u^{m}\right\}$ satisfying that there exists $u \in S\left(\left(u^{\tau}, \psi\right), \tau\right)$ such that

$$
u^{\mu}(t) \rightarrow u(t) \text { weakly in } H, \text { for all } t \geq \tau,
$$

and

$$
u^{\mu} \rightarrow u \text { strongly in } L^{2}\left(\tau, T ; L^{2}\left(\Omega_{n}\right)\right) \text { for all } T>\tau, n \geq 1 .
$$

(ii) If $\left\{\left(u^{\tau, m}, \psi^{m}\right)\right\}$ converges strongly in $\mathcal{H}$ to $\left(u^{\tau}, \psi\right)$, then, there exists a subsequence $\left\{u^{\mu}\right\} \subset\left\{u^{m}\right\}$ satisfying that there exists $u \in S\left(\left(u^{\tau}, \psi\right), \tau\right)$ such that

$$
\begin{gathered}
u^{\mu} \rightarrow u \text { strongly in } L^{2}(\tau, T ; H) \text { for all } T>\tau, \\
u^{\mu}(t) \rightarrow u(t) \text { strongly in } H, \text { for all } t \geq \tau,
\end{gathered}
$$

and in addition, if assumption (H4) is also satisfied, then

$$
u_{T}^{\mu} \rightarrow u_{T} \text { strongly in } L_{V, \lambda_{1}}^{2} \text {, for any } T>\tau .
$$

Proof. If we argue similarly to the proof of Theorem 1 and Lemma 2, we obtain the existence of a subsequence $\left\{u^{\mu}\right\} \subset\left\{u^{m}\right\}$ such that for all $T>\tau$,

$$
u^{\mu} \rightarrow u \text { weakly in } L_{\lambda_{1}}^{2}(-\infty, T ; V), L^{p}\left(\tau, T ; L^{p}(\Omega)\right) \text { and } L^{2}(\tau, T ; V),
$$

$\left\{u^{\mu}(T)\right\}_{\mu \in \mathbb{N}}$ is bounded in $H$, and $u \in S\left(\left(u^{\tau}, \psi\right), \tau\right)$ and satisfies (66).

On the other hand, the boundedness of $\left\{u^{\mu}(T)\right\}_{\mu \in \mathbb{N}}$ in $H$ implies the existence of a subsequence converging weakly in $H$ to some $\xi \in H$. Let $u^{\mu_{k}}(T) \rightarrow \xi$ weakly in $H$. Let $w \in V \cap L^{p}(\Omega)$. From the equation satisfied by $u^{\mu_{k}}$, we obtain

$$
\begin{aligned}
\left(u^{\mu_{k}}(T), w\right)= & \int_{\tau}^{T}\left\langle\Delta u^{\mu_{k}}(t)+K\left(t, u_{t}^{\mu_{k}}\right)-G\left(t, u^{\mu_{k}}(t)\right)+F(t), w\right\rangle d t+\left(u^{\mu_{k}}(\tau), w\right) \\
& \rightarrow(u(\tau), w)+\int_{\tau}^{T}\left\langle\Delta u(t)+K\left(t, u_{t}\right)-G(t, u(t))+F(t), w\right\rangle d t
\end{aligned}
$$

as $\mu_{k} \rightarrow \infty$. Then arguing as in Theorem 1 , we have

$$
(\xi, w)=(u(\tau), w)+\int_{\tau}^{T}\left\langle\Delta u(t)+K\left(t, u_{t}\right)-G(t, u(t))+F(t), w\right\rangle d t .
$$


Consequently, as $u$ is a solution of (5) corresponding to the initial data $(u(\tau), \psi)$, we obtain

$$
(\xi, w)=(u(T), w) \quad \forall w \in V \cap L^{p}(\Omega),
$$

and therefore, by density, it follows

$$
\xi=u(T) .
$$

Then, by a contradiction argument, we can deduce that the whole sequence $\left\{u^{\mu}(T)\right\}_{\mu \in \mathbb{N}}$ converges weakly in $H$ to $u(T)$.

As $T>\tau$ has been taken arbitrarily, we see that (65) holds.

Now we will prove (ii). Let us fix $T>\tau$, and assume that $\left(u^{\tau, m}, \psi^{m}\right) \rightarrow\left(u^{\tau}, \psi\right)$ strongly in $\mathcal{H}$. Then, by Lemma 6 , we have that for all $\varepsilon>0$ there exists $M_{\varepsilon} \geq 1$ such that

$$
\begin{aligned}
\int_{\tau}^{T} \int_{\Omega \cap\left\{|x|_{\mathbb{R}^{N}} \geq 2 n\right\}}\left(u^{m}-u\right)^{2} d x d s & \leq 2 \int_{\tau}^{T} \int_{\Omega \cap\left\{|x|_{\left.\mathbb{R}^{N} \geq 2 n\right\}}\right.}\left(u^{m}\right)^{2} d x d s+2 \int_{\tau}^{T} \int_{\Omega \cap\left\{|x|_{\mathbb{R}^{N}} \geq 2 n\right\}} u^{2} d x d s \\
& \leq 4 \varepsilon(T-\tau), \forall n \geq M_{\varepsilon} .
\end{aligned}
$$

From this inequality and (66) we obtain (67).

From (67) we deduce that from every subsequence of $\left\{u^{\mu}\right\}$ we can extract a subsequence that we will denote by $\left\{u^{\nu}\right\}$ such that

$$
\left|u^{\nu}(t)\right| \rightarrow|u(t)| \text { a.e. in }(\tau, T) .
$$

Let us define

$$
\begin{gathered}
J_{\nu}(t)=\left|u^{\nu}(t)\right|^{2}-\int_{\tau}^{t} c_{4}(s) d s-\int_{\tau}^{t} \frac{2 k}{1-d} e^{-\lambda_{1}(s-\tau)}\left\|\left(u^{\tau, \nu}, \psi^{\nu}\right)\right\|_{\mathcal{H}}^{2} d s-\int_{\tau}^{t} \frac{2 k}{1-d} e^{-\lambda_{1} s} \int_{-\infty}^{s} e^{\lambda_{1} r} c(r) d r d s \\
-\int_{\tau}^{t} \frac{8 k}{1-d} e^{-\lambda_{1} s} \int_{-\infty}^{s} e^{\lambda_{1} r}\|F(r)\|_{-1}^{2} d r d s-2 \int_{\tau}^{t} c_{1}(s) d s-2 \int_{\tau}^{t}\left\langle F(s), u^{\nu}(s)\right\rangle d s,
\end{gathered}
$$

and

$$
\begin{gathered}
J(t)=|u(t)|^{2}-\int_{\tau}^{t} c_{4}(s) d s-\int_{\tau}^{t} \frac{2 k}{1-d} e^{-\lambda_{1}(s-\tau)}\left\|\left(u^{\tau}, \psi\right)\right\|_{\mathcal{H}}^{2} d s-\int_{\tau}^{t} \frac{2 k}{1-d} e^{-\lambda_{1} s} \int_{-\infty}^{s} e^{\lambda_{1} r} c(r) d r d s \\
-\int_{\tau}^{t} \frac{8 k}{1-d} e^{-\lambda_{1} s} \int_{-\infty}^{s} e^{\lambda_{1} r}\|F(r)\|_{-1}^{2} d r d s-2 \int_{\tau}^{t} c_{1}(s) d s-2 \int_{\tau}^{t}\langle F(s), u(s)\rangle d s,
\end{gathered}
$$

for all $t \geq \tau$.

It is clear that $J_{\nu}$ and $J$ are continuous functions. Also, from (70), (71) and the fact that $\left\{\left(u^{\tau, \nu}, \psi^{\nu}\right)\right\}$ converges strongly in $\mathcal{H}$ to $\left(u^{\tau}, \psi\right) \in \mathcal{H}$, we see that

$$
J_{\nu}(t) \rightarrow J(t) \text { a.e. } t \in(\tau, T) \text { as } \nu \rightarrow \infty .
$$

On the other hand, taking into account the energy equality, (6) and (10), we have

$$
\begin{aligned}
\frac{d}{d t}\left|u^{\nu}(t)\right|^{2} & \leq\left\|K\left(t, u_{t}^{\nu}\right)\right\|_{-1}^{2}+2 c_{1}(t)+2\left\langle F(t), u^{\nu}(t)\right\rangle \\
& \leq c_{4}(t)+k\left\|u_{t}^{\nu}\right\|_{L_{V, \lambda_{1}}^{2}}^{2}+2 c_{1}(t)+2\left\langle F(t), u^{\nu}(t)\right\rangle,
\end{aligned}
$$

and using Lemma 1, we obtain

$$
\begin{aligned}
\frac{d}{d t}\left|u^{\nu}(t)\right|^{2} & \leq c_{4}(t)+\frac{2 k}{1-d} e^{-\lambda_{1}(t-\tau)}\left\|\left(u^{\tau, \nu}, \psi^{\nu}\right)\right\|_{\mathcal{H}}^{2}+\frac{2 k}{1-d} e^{-\lambda_{1} t} \int_{-\infty}^{t} e^{\lambda_{1} r} c(r) d r \\
& +\frac{8 k}{1-d} e^{-\lambda_{1} t} \int_{-\infty}^{t} e^{\lambda_{1} r}\|F(r)\|_{-1}^{2} d r+2 c_{1}(t)+2\left\langle F(t), u^{\nu}(t)\right\rangle
\end{aligned}
$$

for all $t>\tau$. Thus, for every $\nu$, the function $J_{\nu}$ is a non-increasing function of $t$.

We are now in a position to show that

$$
J_{\nu}(t) \rightarrow J(t) \text { for all } t \in(\tau, T) .
$$


Let $t \in(\tau, T)$ and $\varepsilon>0$ be fixed. From (72) and the continuity of $J$, we can take $t^{\prime}>t$ and $t^{\prime \prime}<t$ such that

$$
J_{\nu}\left(t^{\prime}\right) \rightarrow J\left(t^{\prime}\right) \quad \text { and } \quad J_{\nu}\left(t^{\prime \prime}\right) \rightarrow J\left(t^{\prime \prime}\right) \quad \text { as } \nu \rightarrow \infty
$$

with

$$
\left|J\left(t^{\prime \prime}\right)-J(t)\right| \leq \varepsilon \quad \text { and } \quad\left|J(t)-J\left(t^{\prime}\right)\right| \leq \varepsilon .
$$

As $J_{\nu}$ is a non-increasing function of $t$, we obtain

$$
J_{\nu}\left(t^{\prime}\right)-J_{\nu}(t) \leq 0 \quad \text { and } \quad J_{\nu}\left(t^{\prime \prime}\right)-J_{\nu}(t) \geq 0,
$$

for every $\nu$.

Using (75) and (76), we have

$$
\begin{aligned}
J_{\nu}(t)-J(t) & =J_{\nu}(t)-J_{\nu}\left(t^{\prime \prime}\right)+J_{\nu}\left(t^{\prime \prime}\right)-J\left(t^{\prime \prime}\right)+J\left(t^{\prime \prime}\right)-J(t) \\
& \leq\left|J_{\nu}\left(t^{\prime \prime}\right)-J\left(t^{\prime \prime}\right)\right|+\varepsilon
\end{aligned}
$$

and

$$
\begin{aligned}
J(t)-J_{\nu}(t) & =J(t)-J\left(t^{\prime}\right)+J\left(t^{\prime}\right)-J_{\nu}\left(t^{\prime}\right)+J_{\nu}\left(t^{\prime}\right)-J_{\nu}(t) \\
& \leq\left|J\left(t^{\prime}\right)-J_{\nu}\left(t^{\prime}\right)\right|+\varepsilon .
\end{aligned}
$$

From (74), (77) and (78), we have

$$
\lim \sup _{\nu \rightarrow \infty}\left|J(t)-J_{\nu}(t)\right| \leq \varepsilon,
$$

and therefore, as $\varepsilon>0$ is arbitrary, (73) follows from (79).

Thanks to (73), and taking into account (70) and that $\left\{\left(u^{\tau, \nu}, \psi^{\nu}\right)\right\}$ converges strongly in $\mathcal{H}$ to $\left(u^{\tau}, \psi\right) \in$ $\mathcal{H}$, we deduce that

$$
\left|u^{\nu}(t)\right| \rightarrow|u(t)| \forall t \in(\tau, T)
$$

and then, by (65), we obtain

$$
u^{\nu}(t) \rightarrow u(t) \text { strongly in } H \quad \forall t \in(\tau, T) .
$$

Then from a standard contradiction argument combined with the fact that $T>\tau$ has been taken arbitrarily, we deduce (68).

Finally, we will prove (69). We observe that the difference $v^{\mu}=u^{\mu}-u$ satisfies

$$
\begin{aligned}
\frac{d}{d t}\left|v^{\mu}(t)\right|^{2}+\lambda_{1}\left|v^{\mu}(t)\right|^{2}+\left|\nabla v^{\mu}(t)\right|^{2} \leq 2 & \left\|K\left(t, u_{t}^{\mu}\right)-K\left(t, u_{t}\right)\right\|_{-1}^{2}+\frac{1}{2}\left|\nabla v^{\mu}(t)\right|^{2} \\
& +2\left\langle F(t), v^{\mu}(t)\right\rangle-2\left\langle G\left(t, u^{\mu}(t)\right)-G(t, u(t)), v^{\mu}(t)\right\rangle,
\end{aligned}
$$

a.e. $t>\tau$.

Multiplying by $e^{\lambda_{1} t}$, integrating between $\tau$ and $T$, and using (13), we obtain

$$
\begin{aligned}
\left|v^{\mu}(T)\right|^{2}+\frac{1-b}{2} \int_{\tau}^{T} e^{-\lambda_{1}(T-s)}\left|\nabla v^{\mu}(s)\right|^{2} d s & \leq e^{-\lambda_{1}(T-\tau)}\left|v^{\mu}(\tau)\right|^{2} \\
& +\frac{b}{2} \int_{-\infty}^{\tau} e^{-\lambda_{1}(T-s)}\left|\nabla v^{\mu}(s)\right|^{2} d s \\
& +2 \int_{\tau}^{T} e^{-\lambda_{1}(T-s)}\left\langle F(s), v^{\mu}(s)\right\rangle d s \\
& -2 \int_{\tau}^{T} e^{-\lambda_{1}(T-s)}\left\langle G\left(s, u^{\mu}(s)\right)-G(s, u(s)), v^{\mu}(s)\right\rangle d s .
\end{aligned}
$$

As $u^{\mu}(\tau) \rightarrow u(\tau)$ strongly in $H$, then

$$
\lim _{\mu \rightarrow \infty} e^{-\lambda_{1}(T-\tau)}\left|v^{\mu}(\tau)\right|^{2}=0
$$


By (70), we have

$$
\lim _{\mu \rightarrow \infty} \int_{\tau}^{T} e^{-\lambda_{1}(T-s)}\left\langle G(s, u(s)), v^{\mu}(s)\right\rangle d s=0
$$

and

$$
\lim _{\mu \rightarrow \infty} \int_{\tau}^{T} e^{-\lambda_{1}(T-s)}\left\langle F(s), v^{\mu}(s)\right\rangle d s=0 .
$$

Also, if we argue similarly to the proof of Theorem 1, we obtain,

$$
G\left(\cdot, u^{\mu}(\cdot)\right) \rightarrow G(\cdot, u(\cdot)) \text { weakly in } L^{q}\left(\tau, T ; L^{q}(\Omega)\right) .
$$

By (67) we know that for every subsequence of $\left\{u^{\mu}\right\}$, there exists a subsequence that we will denote $\left\{u^{\nu}\right\}$ such that $u^{\nu}(t, x) \rightarrow u(t, x)$, a.e. in $(\tau, T) \times \Omega$. Now, we will prove that

$$
\lim \inf _{\nu \rightarrow \infty} \int_{\tau}^{T} e^{-\lambda_{1}(T-s)}\left\langle G\left(s, u^{\nu}(s)\right), u^{\nu}(s)\right\rangle d s \geq \int_{\tau}^{T} e^{-\lambda_{1}(T-s)}\langle G(s, u(s)), u(s)\rangle d s .
$$

The continuity of $g$ implies that $g\left(x, t, u^{\nu}(t, x)\right) \rightarrow g(x, t, u(t, x))$, a.e. in $(\tau, T) \times \Omega$. From (3) we have that

$$
g\left(x, t, u^{\nu}(t, x)\right) u^{\nu}(t, x)+\delta_{1}(x, t) \geq 0,
$$

and then from Lebesgue-Fatou's Lemma (see [Yosida, 1965]) we obtain

$$
\begin{aligned}
& \lim \inf _{\nu \rightarrow \infty} \int_{\tau}^{T} e^{-\lambda_{1}(T-s)}\left\langle G\left(s, u^{\nu}(s)\right), u^{\nu}(s)\right\rangle d s+\int_{\tau}^{T} e^{-\lambda_{1}(T-s)} \int_{\Omega} \delta_{1}(x, s) d x d s \\
& =\lim \inf _{\nu \rightarrow \infty}\left(\int_{\tau}^{T} \int_{\Omega} e^{-\lambda_{1}(T-s)}\left(g\left(x, s, u^{\nu}(s, x)\right) u^{\nu}(s, x)+\delta_{1}(x, s)\right) d x d s\right) \\
& \geq \int_{\tau}^{T} \int_{\Omega} e^{-\lambda_{1}(T-s)} \lim \inf _{\nu \rightarrow \infty}\left(g\left(x, s, u^{\nu}(s, x)\right) u^{\nu}(s, x)+\delta_{1}(x, s)\right) d x d s \\
& =\int_{\tau}^{T} \int_{\Omega} e^{-\lambda_{1}(T-s)}\left(g(x, s, u(s, x)) u(s, x)+\delta_{1}(x, s)\right) d x d s \\
& =\int_{\tau}^{T} e^{-\lambda_{1}(T-s)}\langle G(s, u(s)), u(s)\rangle d s+\int_{\tau}^{T} e^{-\lambda_{1}(T-s)} \int_{\Omega} \delta_{1}(x, s) d x d s,
\end{aligned}
$$

so that (85) holds.

If we use (84) and (85), we have

$$
\begin{aligned}
& \lim \sup _{\nu \rightarrow \infty} \int_{\tau}^{T} e^{-\lambda_{1}(T-s)}\left\langle-G\left(s, u^{\nu}(s)\right), u^{\nu}(s)-u(s)\right\rangle d s \\
& =\int_{\tau}^{T} e^{-\lambda_{1}(T-s)}\langle G(s, u(s)), u(s)\rangle d s \\
& -\lim \inf _{\nu \rightarrow \infty} \int_{\tau}^{T} e^{-\lambda_{1}(T-s)}\left\langle G\left(s, u^{\nu}(s)\right), u^{\nu}(s)\right\rangle d s \leq 0 .
\end{aligned}
$$

Then, taking into account (81)-(83) and (86) in (80), we get

$$
\begin{aligned}
& \lim \sup _{\nu \rightarrow \infty} \int_{\tau}^{T} e^{-\lambda_{1}(T-s)}\left|\nabla v^{\nu}(s)\right|^{2} d s \\
& \leq \frac{b}{1-b} \lim _{\nu \rightarrow \infty} \int_{-\infty}^{\tau} e^{-\lambda_{1}(T-s)}\left|\nabla v^{\nu}(s)\right|^{2} d s \\
& =\frac{b}{1-b} e^{-\lambda_{1}(T-\tau)} \lim _{\nu \rightarrow \infty} \int_{-\infty}^{0} e^{\lambda_{1} r}\left|\nabla \psi^{\nu}(r)-\nabla \psi(r)\right|^{2} d r .
\end{aligned}
$$


From this inequality, observing that

$$
\begin{aligned}
\left\|v_{T}^{\nu}\right\|_{L_{V, \lambda_{1}}^{2}}^{2} & =\int_{\tau-T}^{0} e^{\lambda_{1} s}\left|\nabla v_{T}^{\nu}(s)\right|^{2} d s+\int_{-\infty}^{\tau-T} e^{\lambda_{1} s}\left|\nabla v_{T}^{\nu}(s)\right|^{2} d s \\
& =\int_{\tau}^{T} e^{-\lambda_{1}(T-s)}\left|\nabla v^{\nu}(s)\right|^{2} d s+e^{-\lambda_{1}(T-\tau)} \int_{-\infty}^{0} e^{\lambda_{1} r}\left|\nabla v^{\nu}(r+\tau)\right|^{2} d r \\
& =\int_{\tau}^{T} e^{-\lambda_{1}(T-s)}\left|\nabla v^{\nu}(s)\right|^{2} d s+e^{-\lambda_{1}(T-\tau)} \int_{-\infty}^{0} e^{\lambda_{1} r}\left|\nabla \psi^{\nu}(r)-\nabla \psi(r)\right|^{2} d r,
\end{aligned}
$$

we obtain

$$
\lim \sup _{\nu \rightarrow \infty}\left\|v_{T}^{\nu}\right\|_{L_{V, \lambda_{1}}^{2}}^{2} \leq \frac{1}{1-b} e^{-\lambda_{1}(T-\tau)} \lim \sup _{\nu \rightarrow \infty} \int_{-\infty}^{0} e^{\lambda_{1} r}\left|\nabla \psi^{\nu}(r)-\nabla \psi(r)\right|^{2} d r
$$

and therefore, by the assumption $\psi^{\nu} \rightarrow \psi$ strongly in $L_{V, \lambda_{1}}^{2}$, we deduce that $u_{T}^{\nu} \rightarrow u_{T}$ strongly in $L_{V, \lambda_{1}}^{2}$.

As $\left\{u^{\nu}\right\}$ is a subsequence of an arbitrary subsequence of $\left\{u^{\mu}\right\}$, by a contradiction argument we deduce (69).

Remark 4.2. The results given in Lemma 7 (i) are obtained for the more general case of a continuous operator $G$ satisfying (6), (7) and (H2).

As a consequence of the above result, we obtain that $U$ has compact values.

Corollary 4.1. Under the assumptions in Lemma 5, assume that (H4) also holds. Then, the map $U$ : $\mathbb{R}_{d} \times \mathcal{H} \rightarrow \mathcal{P}(\mathcal{H})$ defined by (40), has compact values, that is, $U(t, s, x)$ is a compact set for all $(t, s, x)$.

Proof. Thanks to Lemma 7, we obtain that for every $t \geq \tau$, and any $\left(u^{\tau}, \psi\right) \in \mathcal{H}$, the set $U\left(t, \tau,\left(u^{\tau}, \psi\right)\right)$ is relatively compact and closed in $H \times L_{V, \lambda_{1}}^{2}$.

\subsection{Existence of pullback attractors}

Now, we are ready to obtain the existence of pullback attractors for the MNDS $U$ defined by (40).

Lemma 8. Under the assumptions in Lemma 5, assume that (H4) also holds. Then, the MNDS U defined by (40) is pullback asymptotically compact with respect to the family $\widehat{B}_{\lambda_{1}}$ defined in Lemma 4.

Proof. Let us fix $t \in \mathbb{R}$, a sequence $\tau_{m} \leq t$ with $\tau_{m} \rightarrow-\infty$, and a sequence $\left(u^{\tau_{m}}, \psi^{m}\right) \in B_{\lambda_{1}}\left(\tau_{m}\right)$. We have to prove that from any sequence $\left(z^{m}, y^{m}\right) \in U\left(t, \tau_{m},\left(u^{\tau_{m}}, \psi^{m}\right)\right)$ we can extract a subsequence that converges strongly in $\mathcal{H}$.

As $\left(z^{m}, y^{m}\right) \in U\left(t, \tau_{m},\left(u^{\tau_{m}}, \psi^{m}\right)\right)$, there exists $u^{m} \in S\left(\left(u^{\tau_{m}}, \psi^{m}\right), \tau_{m}\right)$ such that $u^{m}(t)=z^{m}$ and $u_{t}^{m}=y^{m}$.

Fixing $k \geq 1$, as the family $\widehat{B}_{\lambda_{1}}$ belongs to $\mathcal{D}_{\lambda_{1}}$, is pullback $\mathcal{D}_{\lambda_{1}}$-absorbing, and $\tau_{m} \rightarrow-\infty$, there exists $m_{k}(t) \geq k$ such that $\tau_{m} \leq t-k$ and

$$
\begin{aligned}
\left(u^{m}(t-k), u_{t-k}^{m}\right) & \in U\left(t-k, \tau_{m},\left(u^{\tau_{m}}, \psi^{m}\right)\right) \\
& \subset U\left(t-k, \tau_{m}, B_{\lambda_{1}}\left(\tau_{m}\right)\right) \subset B_{\lambda_{1}}(t-k),
\end{aligned}
$$

for all $m \geq m_{k}(t)$. Thus,

$$
\begin{aligned}
U\left(t, \tau_{m},\left(u^{\tau_{m}}, \psi^{m}\right)\right) & =U\left(t, t-k, U\left(t-k, \tau_{m},\left(u^{\tau_{m}}, \psi^{m}\right)\right)\right) \\
& \subset U\left(t, t-k, B_{\lambda_{1}}(t-k)\right),
\end{aligned}
$$

for all $m \geq m_{k}(t)$.

This implies that $\left(z^{m}, y^{m}\right) \in U\left(t, t-k,\left(\zeta^{m, k}, \xi^{m, k}\right)\right)$ where $\left(\zeta^{m, k}, \xi^{m, k}\right) \in B_{\lambda_{1}}(t-k)$, with $u^{m}(t-k)=\zeta^{m, k}$ and $u_{t-k}^{m}=\xi^{m, k}$. 
Since $B_{\lambda_{1}}(t-k)$ is a bounded closed ball in $\mathcal{H}$, we can assume, up to a subsequence, that, in particular, $\xi^{m, k} \rightarrow \xi^{k}$ weakly in $L_{V, \lambda_{1}}^{2}$,

and

$$
\zeta^{m, k} \rightarrow \zeta^{k} \text { weakly in } H
$$

where $\left(\zeta^{k}, \xi^{k}\right) \in B_{\lambda_{1}}(t-k)$.

Then, as $u^{m} \in S\left(\left(u^{m}(t-k), u_{t-k}^{m}\right), t-k\right)$, by Lemma 7 (i), we have that there exists a subsequence $\left\{u^{m^{\prime}, k}\right\} \subset\left\{u^{m}\right\}$ such that there exists $u \in S\left(\left(\zeta^{k}, \xi^{k}\right), t-k\right)$ satisfying

$$
z^{m^{\prime}, k}=u^{m^{\prime}, k}(t) \rightarrow u(t) \text { weakly in } H,
$$

and

$$
u^{m^{\prime}, k} \rightarrow u \text { strongly in } L^{2}\left(t-k, t ; L^{2}\left(\Omega_{2 n}\right)\right) \text {, for } n \geq 1 .
$$

as $m^{\prime} \rightarrow \infty$.

By Lemma 5, and thanks to the fact that $\left(u^{m}(t-k), u_{t-k}^{m}\right) \in B_{\lambda_{1}}(t-k)$, for any $\varepsilon>0$, there exists $T\left(t-k, t, \varepsilon, \widehat{B}_{\lambda_{1}}\right) \leq t-k$, and $M_{1}\left(t-k, t, \varepsilon, \widehat{B}_{\lambda_{1}}\right) \geq 1$, such that

$$
\int_{t-k}^{t} \int_{|x|_{\mathbb{R}^{N}} \geq 2 n}\left(u^{m}\right)^{2}(x, s) d x d s \leq k \varepsilon,
$$

for all $n \geq M_{1}\left(t-k, t, \varepsilon, \widehat{B}_{\lambda_{1}}\right)$ and any $m$ such that $\tau_{m} \leq T\left(t-k, t, \varepsilon, \widehat{B}_{\lambda_{1}}\right)$.

On the other hand, by Lemma 6 , for any $\varepsilon>0$ there exists $M_{2}(t-k, t, \varepsilon)$, such that

$$
\int_{t-k}^{t} \int_{|x|_{\mathbb{R}^{N}} \geq 2 n} u^{2}(x, s) d x d s \leq k \varepsilon,
$$

for all $n \geq M_{2}(t-k, t, \varepsilon)$.

Then, we deduce that

$$
\int_{t-k}^{t} \int_{|x|_{\mathbb{R}^{N}} \geq 2 n}\left(u^{m}(x, s)-u(x, s)\right)^{2} d x d s \leq 4 k \varepsilon,
$$

for all $n \geq \max \left\{M_{1}\left(t-k, t, \varepsilon, \widehat{B}_{\lambda_{1}}\right), M_{2}(t-k, t, \varepsilon)\right\}$, and any $m$ such that $\tau_{m} \leq T\left(t-k, t, \varepsilon, \widehat{B}_{\lambda_{1}}\right)$. From (88) and (89) we have

$$
u^{m^{\prime}, k} \rightarrow u \text { strongly in } L^{2}(t-k, t ; H),
$$

as $m^{\prime} \rightarrow \infty$.

Now, if we argue similarly to the proof of Lemma 7 , but using the functional $J$ with $\frac{2 k R_{\lambda_{1}}(t-k)}{\lambda_{1}(1-d)}\left[1-e^{-\lambda_{1}(t-\tau)}\right]$ instead of $\int_{\tau}^{t} \frac{2 k}{1-d} e^{-\lambda_{1}(s-\tau)}\left\|\left(u^{\tau}, \psi\right)\right\|_{\mathcal{H}}^{2} d s$ and a similar change for the functional $J_{m^{\prime}, k}$, we obtain

$$
z^{m^{\prime}, k}=u^{m^{\prime}, k}(t) \rightarrow u(t) \text { strongly in } H,
$$

as $m^{\prime} \rightarrow \infty$.

Now, observe that as $\left(u(t), u_{t}\right) \in U\left(t, t-k,\left(\zeta^{k}, \xi^{k}\right)\right)$, then, reasoning as for the obtention of (87) in the proof of Lemma 7 (ii), and taking into account that $\left(\zeta^{m, k}, \xi^{m, k}\right)$ and $\left(\zeta^{k}, \xi^{k}\right)$ belong to $B_{\lambda_{1}}(t-k)$, we obtain

$$
\begin{aligned}
\limsup _{m^{\prime} \rightarrow \infty}\left\|u_{t}^{m^{\prime}, k}-u_{t}\right\|_{L_{V, \lambda_{1}}^{2}}^{2} & \leq \frac{2 e^{-\lambda_{1} k}}{1-b} \limsup _{m^{\prime} \rightarrow \infty}\left(\left|\zeta^{m^{\prime}, k}-\zeta^{k}\right|^{2}+\left\|\xi^{m^{\prime}, k}-\xi^{k}\right\|_{L_{V, \lambda_{1}}^{2}}^{2}\right) \\
& \leq \frac{8}{1-b} e^{-\lambda_{1} k} R_{\lambda_{1}}^{2}(t-k) .
\end{aligned}
$$


Using (90), (91), and the fact that $e^{-\lambda_{1} k} R_{\lambda_{1}}^{2}(t-k) \rightarrow 0$ as $k \rightarrow \infty$, it is not difficult to deduce, via a diagonal procedure, the existence of a subsequence $u^{m^{\prime \prime}}$ such that $u^{m^{\prime \prime}}(t)$ converges to $u(t)$ strongly in $H$, and $u_{t}^{m^{\prime \prime}}$ converges strongly to $u_{t}$ in $L_{V, \lambda_{1}}^{2}$.

Lemma 9. Under the assumptions in Lemma 8, the map $\left(u^{\tau}, \psi\right) \mapsto U\left(t, \tau,\left(u^{\tau}, \psi\right)\right)$ defined by (40) is upper semicontinuous for any $(t, \tau) \in \mathbb{R}_{d}^{2}$.

Proof. If $U$ is not upper semicontinuous, then there exist $\tau \leq t,\left(u^{\tau}, \psi\right) \in \mathcal{H}$, a neighborhood $\mathcal{N}$ of $U\left(t, \tau,\left(u^{\tau}, \psi\right)\right)$ and a sequence $\left(\zeta^{m}, \xi^{m}\right) \in U\left(t, \tau,\left(u^{\tau, m}, \psi^{m}\right)\right)$, where $\left(u^{\tau, m}, \psi^{m}\right) \rightarrow\left(u^{\tau}, \psi\right)$ strongly in $\mathcal{H}$, such that $\left(\zeta^{m}, \xi^{m}\right) \notin \mathcal{N}$. Lemma 7 (ii) implies that there exist subsequences $\left\{\zeta^{\mu}\right\} \subset\left\{\zeta^{m}\right\},\left\{\xi^{\mu}\right\} \subset\left\{\xi^{m}\right\}$ and $(\zeta, \xi) \in U\left(t, \tau,\left(u^{\tau}, \psi\right)\right)$ such that $\zeta^{\mu} \rightarrow \zeta$ strongly in $L^{2}(\Omega)$ and $\xi^{\mu} \rightarrow \xi$ strongly in $L_{V, \lambda_{1}}^{2}$, which is a contradiction.

Now, as a consequence of the preceding results, we can deduce the existence of pullback attractors for the MNDS $U$ defined by (40).

Theorem 3. Assume that $G$ is given by (16), with $g:(x, t) \in \Omega \times \mathbb{R} \mapsto g(x, t, r) \in \mathbb{R}$ measurable for all $r \in \mathbb{R}, g(x, t, \cdot) \in C(\mathbb{R})$ for a.e. $(x, t) \in \Omega \times \mathbb{R}$, satisfying (3) and (4). Suppose also that assumptions $(H 1)$ and $(H 3)-(H 6)$ are satisfied, and that (41) holds, with $c_{1}(\cdot)=\int_{\Omega} \delta_{1}(x, \cdot) d x$. Then, the MNDSU defined by (40) possesses a unique pullback $\mathcal{D}_{\lambda_{1}}$ - attractor $\mathcal{A}_{\mathcal{D}_{\lambda_{1}}}$ belonging to $\mathcal{D}_{\lambda_{1}}$, which is strictly invariant and is given by

$$
\mathcal{A}_{\mathcal{D}_{\lambda_{1}}}(t)=\Lambda\left(\widehat{B}_{\lambda_{1}}, t\right)=\bigcap_{s \leq t \tau \leq s} \overline{\bigcup U\left(t, \tau, B_{\lambda_{1}}(\tau)\right)}
$$

where $\widehat{B}_{\lambda_{1}}$ was defined in Lemma 4 , and the closure is taken in $\mathcal{H}$. Moreover, we have the following relation

$$
\mathcal{A}_{\mathcal{D}_{\lambda_{1}}}(t) \subset \bar{B}_{\mathcal{H}}\left(0, R_{\lambda_{1}}(t)\right) \quad \text { for all } t \in \mathbb{R} \text {. }
$$

Remark 4.3. Observe that the universe $\mathcal{D}_{\lambda_{1}}$ contains the families of fixed bounded sets (i.e. for any bounded $C \subset \mathcal{H}$ it follows that $\left.\widehat{C}=\{C(t) \equiv C, t \in \mathbb{R}\} \in \mathcal{D}_{\lambda_{1}}\right)$. It is easy to conclude, under the assumptions of Theorem 3, the existence of the pullback attractor $\mathcal{A}_{\mathcal{D}_{F}^{\mathcal{H}}}$ in the sense of Crauel et al. [1997] and the following relation:

$$
\mathcal{A}_{\mathcal{D}_{F}^{\mathcal{H}}}(t) \subset \mathcal{A}_{\mathcal{D}_{\lambda_{1}}}(t) \text { for any } t \in \mathbb{R} .
$$

In fact, it can be proved (see [Marín-Rubio \& Real, 2009]) that if there exists a value $T \in \mathbb{R}$ such that

$$
\sup _{t \leq T} R_{\lambda_{1}}(t)<+\infty
$$

where $R_{\lambda_{1}}$ is the function defined in (43), then

$$
\mathcal{A}_{\mathcal{D}_{F}^{\mathcal{H}}}(t)=\mathcal{A}_{\mathcal{D}_{\lambda_{1}}}(t) \quad \forall t \leq T .
$$

\section{Application to equation (2)}

In this section, we analyze our example (2). We will check that under adequate assumptions on the functions appearing in this equation all the hypotheses established for the abstract equation are fulfilled in this particular case.

First, if we define $F(t)=f(t)$ where $f \in L_{l o c}^{2}\left(\mathbb{R} ; H^{-1}(\Omega)\right)$ is such that

$$
\int_{-\infty}^{t} e^{\lambda_{1} s}\|f(s)\|_{-1}^{2} d s<+\infty \quad \forall t \in \mathbb{R}
$$

then $(H 6)$ holds.

On the other hand, if we define $G$ by (16), with $g:(x, t) \in \Omega \times \mathbb{R} \mapsto g(x, t, r) \in \mathbb{R}$ measurable for all $r \in \mathbb{R}, g(x, t, \cdot) \in C(\mathbb{R})$ for a.e. $(x, t) \in \Omega \times \mathbb{R}$, satisfying (3) and (4), then it is evident that $G$ is a well defined measurable mapping from $\mathbb{R} \times L^{p}(\Omega)$ into $L^{q}(\Omega)$, and satisfies (6) and (7). On the other hand, 
using Lebesgue's Theorem, it is not difficult to prove that for a.e $t \in \mathbb{R}, G(t, \cdot)$ is continuous from $L^{p}(\Omega)$ into $L^{q}(\Omega)$.

Now, we will prove $(H 2)$. For this, we consider a sequence $\left\{u^{m}\right\}_{m \in \mathbb{N}}$ such that $u^{m} \rightarrow u$ strongly in $L^{2}\left(\tau, T ; L^{2}\left(\Omega_{n}\right)\right)$ and $u^{m} \rightarrow u$ weakly in $L^{p}\left(\tau, T ; L^{p}(\Omega)\right)$. By $u^{m} \rightarrow u$ strongly in $L^{2}\left(\tau, T ; L^{2}\left(\Omega_{n}\right)\right)$ we know that there exists a subsequence of $\left\{u^{m}\right\}$, denoted also by $\left\{u^{m}\right\}$, such that

$$
u^{m}(t, x) \rightarrow u(t, x) \text { for a.e. }(t, x) \in(\tau, T) \times \Omega_{n} .
$$

Hence, the continuity of the map $v \mapsto g(x, t, v)$ implies that

$$
g\left(x, t, u^{m}(t, x)\right) \rightarrow g(x, t, u(t, x)) \text { for a.e. }(t, x) \in(\tau, T) \times \Omega_{n} .
$$

From (7), we have

$$
\left\|G\left(\cdot, u^{m}(\cdot)\right)\right\|_{L^{q}\left(\tau, T ; L^{q}(\Omega)\right)}^{q} \leq \int_{\tau}^{T}\left(\rho\left|u^{m}(t)\right|_{p}^{p}+c_{2}(t)\right) d t,
$$

and, as $u^{m} \rightarrow u$ weakly in $L^{p}\left(\tau, T ; L^{p}(\Omega)\right)$ and $c_{2} \in L_{l o c}^{1}(\mathbb{R})$, in particular, we have

$$
G\left(\cdot, u^{m}(\cdot)\right) \text { is bounded in } L^{q}\left(\tau, T ; L^{q}\left(\Omega_{n}\right)\right) .
$$

From (94), (95), and by Lemma 1.3, Chapter 1 in [Lions, 1969], we obtain

$$
G\left(\cdot, u^{m}(\cdot)\right) \rightarrow G(\cdot, u(\cdot)) \text { weakly in } L^{q}\left(\tau, T ; L^{q}\left(\Omega_{n}\right)\right) .
$$

Therefore, (11) is satisfied.

On the other hand, let us define $K: \mathbb{R} \times L_{V, \lambda_{1}}^{2} \rightarrow V^{\prime}$ as

$$
\begin{aligned}
\langle K(t, \psi), v\rangle & =-\int_{-\infty}^{t} \gamma(t-s)\langle\Delta \psi(x, s-t), v\rangle d s \\
& =\int_{-\infty}^{t} \gamma(t-s)(\nabla \psi(s-t), \nabla v) d s,
\end{aligned}
$$

for $v \in V$, where the function $\gamma \in L^{1}\left(\mathbb{R}_{+}\right)$satisfies

$$
|\gamma(\theta)| \leq \gamma_{0} e^{-d_{0} \theta} \text {, a.e. } \theta>0,
$$

for some constants

$$
\gamma_{0}>0 \text { and } d_{0}>\lambda_{1}
$$

such that

$$
\frac{8 \gamma_{0}^{2}}{d_{0}\left(d_{0}-\lambda_{1}\right)}<1
$$

Then,

$$
\begin{aligned}
\|K(t, \psi)\|_{-1}=\sup _{|\nabla v| \leq 1}|\langle K(t, \psi), v\rangle| & \leq \int_{-\infty}^{t} \gamma_{0} e^{-d_{0}(t-s)}|\nabla \psi(s-t)| d s \\
& =\int_{-\infty}^{0} \gamma_{0} e^{d_{0} s}|\nabla \psi(s)| d s \\
& \leq\left(\int_{-\infty}^{0} \gamma_{0}^{2} e^{\left(2 d_{0}-\lambda_{1}\right) s} d s\right)^{1 / 2}\|\psi\|_{L_{V, \lambda_{1}}^{2}} \\
& =\frac{\gamma_{0}}{\left(2 d_{0}-\lambda_{1}\right)^{1 / 2}}\|\psi\|_{L_{V, \lambda_{1}}^{2}} .
\end{aligned}
$$

Therefore, (10) holds with $k=\gamma_{0}^{2} /\left(2 d_{0}-\lambda_{1}\right)$ and $c_{4} \equiv 0$. 
Moreover, for $t>\tau$ and $u \in L_{\lambda_{1}}^{2}(-\infty, t ; V)$,

$$
\begin{aligned}
\int_{\tau}^{t} e^{\lambda_{1} s}\left\|K\left(s, u_{s}\right)\right\|_{-1}^{2} d s & \leq \int_{\tau}^{t} e^{\lambda_{1} s}\left(\int_{-\infty}^{s} \gamma_{0} e^{-d_{0}(s-r)}|\nabla u(r)| d r\right)^{2} d s \\
& \leq \int_{\tau}^{t} e^{\lambda_{1} s}\left(\int_{-\infty}^{s} \gamma_{0}^{2} e^{-d_{0}(s-r)} d r\right)\left(\int_{-\infty}^{s} e^{-d_{0}(s-r)}|\nabla u(r)|^{2} d r\right) d s \\
& =\frac{\gamma_{0}^{2}}{d_{0}} \int_{\tau}^{t} e^{\lambda_{1} s}\left(\int_{-\infty}^{s} e^{-d_{0}(s-r)}|\nabla u(r)|^{2} d r\right) d s \\
& \leq \frac{\gamma_{0}^{2}}{d_{0}} \int_{-\infty}^{t}\left(\int_{r}^{t} e^{\lambda_{1} s} e^{-d_{0}(s-r)}|\nabla u(r)|^{2} d s\right) d r \\
& \leq \frac{\gamma_{0}^{2}}{d_{0}\left(d_{0}-\lambda_{1}\right)} \int_{-\infty}^{t} e^{\lambda_{1} r}|\nabla u(r)|^{2} d r,
\end{aligned}
$$

in view of (99) and (9) holds with $d=8 \gamma_{0}^{2} /\left[d_{0}\left(d_{0}-\lambda_{1}\right)\right]$ and $c_{3} \equiv 0$.

It is clear from the above estimates and the linearity of $K(t, \cdot)$ defined by (96) that for all $t \in \mathbb{R}$ the mapping $K(t, \cdot): L_{V, \lambda_{1}}^{2} \rightarrow V^{\prime}$ is continuous, and (H4) holds, with $b=d / 2$. Also, from the above inequality, we see that $(H 5)$ is satisfied.

Finally, assume that $u^{m} \rightarrow u$ weakly in $L_{\lambda_{1}}^{2}(-\infty, T ; V)$. We note that for any $\psi \in L^{2}(\tau, T ; V)$,

$$
\begin{aligned}
\int_{\tau}^{T}\left\langle K\left(s, u_{s}^{m}\right), \psi(s)\right\rangle d s & =\int_{\tau}^{T} \int_{-\infty}^{s} \gamma(s-r)\left(\nabla u^{m}(r), \nabla \psi(s)\right) d r d s \\
& =\int_{\tau}^{T} \int_{-\infty}^{0} \gamma(-\theta)\left(\nabla u^{m}(\theta+s), \nabla \psi(s)\right) d \theta d s \\
& =\int_{\tau}^{T} \int_{-\infty}^{0} e^{\lambda_{1} \theta}\left(\nabla u^{m}(\theta+s), e^{-\lambda_{1} \theta} \gamma(-\theta) \nabla \psi(s)\right) d \theta d s .
\end{aligned}
$$

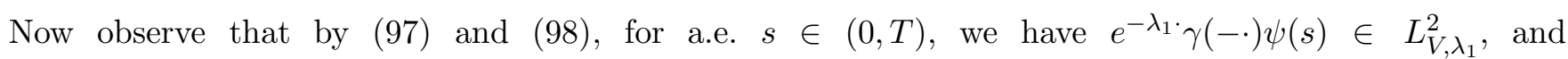
$u^{m}(\cdot+s) \rightarrow u(\cdot+s)$ weakly in $L_{V, \lambda_{1}}^{2}$. Thus,

$$
\int_{-\infty}^{0} e^{\lambda_{1} \theta}\left(\nabla u^{m}(\theta+s), e^{-\lambda_{1} \theta} \gamma(-\theta) \nabla \psi(s)\right) d \theta \rightarrow \int_{-\infty}^{0} e^{\lambda_{1} \theta}\left(\nabla u(\theta+s), e^{-\lambda_{1} \theta} \gamma(-\theta) \nabla \psi(s)\right) d \theta,
$$

as $m \rightarrow \infty$, for a.e. $s \in(0, T)$.

Also, by the boundedness of $u^{m}$ in $L_{\lambda_{1}}^{2}(-\infty, T ; V)$, we have

$$
\begin{aligned}
\left\|u_{s}^{m}\right\|_{L_{V, \lambda_{1}}^{2}}^{2} & =\int_{-\infty}^{0} e^{\lambda_{1} r}\left|\nabla u^{m}(s+r)\right|^{2} d r=\int_{-\infty}^{s} e^{\lambda_{1}(\theta-s)}\left|\nabla u^{m}(\theta)\right|^{2} d \theta \\
& \leq e^{-\lambda_{1} \tau} \int_{-\infty}^{T} e^{\lambda_{1} \theta}\left|\nabla u^{m}(\theta)\right|^{2} d \theta \leq C e^{-\lambda_{1} \tau}
\end{aligned}
$$

for a.e. $s \in(\tau, T)$, and therefore,

$$
\left|\left\langle K\left(s, u_{s}^{m}\right), \psi(s)\right\rangle\right| \leq\left\|K\left(s, u_{s}^{m}\right)\right\|_{-1}|\nabla \psi(s)| \leq \frac{\gamma_{0}}{\left(2 d_{0}-\lambda_{1}\right)^{1 / 2}} C^{1 / 2} e^{-\lambda_{1} \tau / 2}|\nabla \psi(s)| .
$$

Thus, by (100) and Lebesgue's Theorem, we obtain that

$$
\int_{\tau}^{T}\left\langle K\left(s, u_{s}^{m}\right), \psi(s)\right\rangle d s \rightarrow \int_{\tau}^{T}\left\langle K\left(s, u_{s}\right), \psi(s)\right\rangle d s
$$

as $m \rightarrow \infty$, and so (H3) holds.

Now, we apply Theorem 3 and Remark 4.3 for the equation (2), and obtain the following result. 
Theorem 4. Assume that $G$ is given by (16), with $g:(x, t) \in \Omega \times \mathbb{R} \mapsto g(x, t, r) \in \mathbb{R}$ measurable for all $r \in \mathbb{R}, g(x, t, \cdot) \in C(\mathbb{R})$ for a.e. $(x, t) \in \Omega \times \mathbb{R}$, satisfying (3) and (4), and that (41) holds, with $c_{1}(\cdot)=\int_{\Omega} \delta_{1}(x, \cdot) d x$. Suppose that $F=f \in L_{\text {loc }}^{2}\left(\mathbb{R} ; V^{\prime}\right)$ satisfies (93), and $K$ is given by (96), where $\gamma \in L^{1}\left(\mathbb{R}_{+}\right)$is such that conditions (97)-(99) are satisfied. Then, the MNDS defined in this case by (40) (i.e., by equation (2) with Dirichlet boundary conditions) possesses a unique pullback $\mathcal{D}_{\lambda_{1}}$-attractor $\mathcal{A}_{\mathcal{D}_{\lambda_{1}}}$ belonging to $\mathcal{D}_{\lambda_{1}}$, which is strictly invariant and is given by (92). Moreover, there exists the pullback attractor $\mathcal{A}_{\mathcal{D}_{F}^{\mathcal{H}}}$ in the sense of Crauel et al. [1997], and we have the following relation

$$
\mathcal{A}_{\mathcal{D}_{F}^{\mathcal{H}}}(t) \subset \mathcal{A}_{\mathcal{D}_{\lambda_{1}}}(t) \subset \bar{B}_{\mathcal{H}}\left(0, R_{\lambda_{1}}(t)\right) \quad \text { for all } t \in \mathbb{R},
$$

where $R_{\lambda_{1}}(t)$ is given by (43).

Acknowledgement. We would like to thank the referees for their comments and suggestions which allowed us to improve the presentation of this paper.

This work has been partially supported by FEDER and Ministerio de Ciencia e Innovación grant \# MTM2011-22411. Spain.

\section{References}

Anguiano, M. [2011] Atractores para EDP parabólicas no lineales y no autónomas en dominios no acotados, Ph.D.Thesis, Universidad de Sevilla, Sevilla.

Anguiano, M., Caraballo, T., Real, J. \& Valero, J. [2010] "Pullback attractors for reaction-diffusion equations in some unbounded domains with an $H^{-1}$-valued non-autonomous forcing term and without uniqueness of solutions," Discrete Continuous Dynamical Systems, Series B, 14, no. 2, 307-326.

Anguiano, M., Morillas, F. \& Valero, J. [2012] "On the Kneser property for reaction-diffusion equations in some unbounded domains with an $H^{-1}$-valued non-autonomous forcing term", Nonlinear Analysis, 75, 2623-2636.

Brezis, H. [1983] Analyse Fonctionnelle. Thorie et Applications, Masson, Paris.

Caraballo, T., Chueshov, I.D. \& Real, J. [2008] "Pullback attractors for stochastic heat equations in materials with memory", Discrete Contin. Dyn. Syst. Ser. B, 9, 525-539.

Caraballo, T., Garrido-Atienza, M.J., Schmalfuß B. \& Valero, J. [2010] "Global attractor for a nonautonomous integro-differential equation in materials with memory", Nonlinear Analysis, 73, no. $1,183-201$.

Caraballo, T. \& Kloeden, P.E. [2009] "Non-autonomous attractors for integro-differential evolution equations", Discrete Contin. Dyn. Syst. Ser. S, 2, no. 1, 17-36.

Chepyzhov, V.V., Gatti, S., Grasselli, M., Miranville, A. \& Pata, V. [2006] "Trajectory and global attractors for evolutions equations with memory", Appl. Math. Lett., 19, 87-96.

Chepyzhov, V.V., Mainini, E. \& Pata, V. [2006] "Stability of abstract linear semigroups arising from heat conduction with memory", Asymptot. Anal., 50, no. 3-4, 269-291.

Chepyzhov, V.V. \& Miranville, A. [2006] "On trajectory and global attractors for semilinear heat equations with fading memory", Indiana Univ. Math. J., 55, no. 1, 119-167.

Crauel, H., Debussche, A. \& Flandoli, F. [1997] "Random attractors", J. Dynam. Differential Equations, 9, no. 2, 307-341.

Fabrizio, M. \& Morro, A. [1992] Mathematical Problems in Linear Viscoelasticity, SIAM Studies in Applied Mathematics, 12, SIAM, Philadelphia.

Gatti, S., Grasselli, M. \& Pata, V. [2005] "Lyapunov functionals for reaction-diffusion equations with memory", Math. Methods Appl. Sci., 28, no. 14, 1725-1735.

Hino, Y., Murakami, S. \& Naito, T. [1991] Functional Differential Equations with Infinite Delay. Lecture Notes in Mathematics, vol. 1473, Springer-Verlag, Berlin.

Lions, J.L. [1969] Quelques Méthodes de Résolution des Problèmes aux Limites Non Linéaires. Dunod, Paris.

Marín-Rubio, P. \& Real, J. [2009] "On the relation between two different concepts of pullback attractors for non-autonomous dynamical systems", Nonlinear Analysis, 71, 3956-3963. 
Marín-Rubio, P. \& Real, J. [2010] "Pullback attractors for 2D-Navier-Stokes equations with delays in continuous and sub-linear operators", Discrete Contin. Dyn. Syst., 26, 989-1006.

Melnik, V.S. \& Valero, J. [1998] "On attractors of multivalued semi-flows and differential inclusions", Set-Valued Anal., 6, 83-111.

Renardy, M., Hrusa, W.J. \& Nohel, J.A. [1987] Mathematical Problems in Viscoelasticity, Longman, Harlow. John Wiley, New York.

Temam, R. [1983] Navier-Stokes Equations and Nonlinear Functional Analysis. In "CBMS-NSF Regional Conference Series in Applied Mathematics," Vol. 66, SIAM, Philadelphia (2nd edition, 1995).

Wang, L. \& Xu, D. [2003] "Asymptotic behavior of a class of reaction-diffusion equations with delays", $J$. Math. Anal. Appl., 281, 439-453.

Yosida, K. [1965] Functional Analysis, Springer-Verlag, Berlín, Heidelberg. 